Supporting Information for

\title{
Self-Consistent Polarization of the Boundary in the Redistributed Charge and Dipole Scheme for Combined Quantum Mechanical and Molecular Mechanical Calculations
}

\begin{abstract}
Yan Zhang, ${ }^{\dagger}$ Hai Lin, ${ }^{* \dagger}{ }^{\dagger}$ and Donald G. Truhlar ${ }^{\dagger}$
Chemistry Department, University of Colorado at Denver and Health Sciences Center, Denver, Colorado 80217- 3364 and Chemistry Department and Supercomputing Institute, University of Minnesota, Minneapolis, Minnesota 55455-0431
\end{abstract}

${ }^{\dagger}$ University of Colorado at Denver and Heath Science Center

‡ University of Minnesota

*Email: hai.lin@cudenver.edu

Preparation Date: April 8, 2007 


\section{List of Content}

Table S1. The QEq and EEM parameters implemented in the QMMM program

Table S2. Additional MM parameters in the TINKER parameter file format .5

Table S3. Atom types, full-QM optimized coordinates (at the HF/MIDI! level), and connectivities for the amino acids in the tinker .xyz file format 8

Table S4. Atomic charges derived from the full-QM and selected QM/MM calculations (with $\mathrm{QM}=\mathrm{HF} / \mathrm{MIDI} !)$ for the amino acids

Table S5. The convergence of the atomic charges of the heavy atoms in the PS and SS and the convergence of embedded-QM (with QM = HF/MIDI!) energies for the CPS in the PBRC calculations employing the QEq-SCT model for the Ace-His ${ }^{+}$-NMe system and its deprotonated forms

Table S6. Comparison of the charges on H11, H21 and N15, the distance between H11 and H21, and the distance between $\mathrm{H} 11$ and N15 for the Ace-His ${ }^{+}-\mathrm{NMe}$ system and its deprotonated forms (with $\mathrm{QM}=\mathrm{HF} / \mathrm{MIDI} !)$

Table S7. Atomic charges (in the unit of e) derived from the full-QM and selected QM/MM calculations for the amino acids at the full-QM optimized geometries at the HF/MIDI! level.....28

Table S8. Atom types, full-QM optimized coordinates (at the B3LYP/6-31+G* level), and connectivities for the amino acids in the tinker .xyz file format

Table S9. Full-QM and QM/MM proton affinities for the small organic molecules (with QM = B3LYP/6-31+G*)

Table S10. QM/MM optimized Q1-M1 bond distances $(\AA)$ in comparison with full-QM results for small organic molecules (with $\mathrm{QM}=\mathrm{B} 3 \mathrm{LYP} / 6-31+\mathrm{G}^{*}$ ) 
Table S11. Full-QM and QM/MM proton affinities for the small organic molecules (with QM = $\left.\mathrm{MP} 2 / 6-31+\mathrm{G}^{*}\right)$.

Table S12. QM/MM optimized Q1-M1 bond distances $(\AA)$ in comparison with full-QM results for small organic molecules $\left(\mathrm{QM}=\mathrm{MP} 2 / 6-31+\mathrm{G}^{*}\right)$

Table S13. Full-QM and QM/MM proton affinities for amino acids (with $\mathrm{QM}=\mathrm{B} 3 \mathrm{LYP} / 6$ -

$\left.31+\mathrm{G}^{*}\right)$

Table S14. QM/MM optimized Q1-M1 bond distances $(\AA)$ in comparison with full-QM results for amino acids (with $\mathrm{QM}=\mathrm{B} 3 \mathrm{LYP} / 6-31+\mathrm{G}^{*}$ ).

Table S15. Single-point full-QM and QM/MM proton affinities for amino acids at the full-QM optimized geometries for the amino acids (with $\mathrm{QM}=\mathrm{B} 3 \mathrm{LYP} / 6-31+\mathrm{G}^{*}$ ).

Table S16. Full-QM and QM/MM proton affinities $(\mathrm{kcal} / \mathrm{mol})$ for amino acids (with $\mathrm{QM}=$ $\left.\mathrm{MP} 2 / 6-31+\mathrm{G}^{*}\right)$ .58

Table S17. Single-point QM and QM/MM (with QM = MP2/6-31+G*) proton affinities ( $\mathrm{kcal} / \mathrm{mol}$ ) for amino acids at the B3LYP/6-31+G* full-QM optimized geometries

Table S18. QM and QM/MM (with $\mathrm{QM}=\mathrm{B} 3 \mathrm{LYP} / 6-31+\mathrm{G}^{*}$ ) atomic charges (in the unit of e) for Ace-His ${ }^{\delta}-\mathrm{NMe}$

Table S19. Single-point QM and QM/MM (with QM = MP2/6-31+G*) atomic charges for AceHis ${ }^{\delta}-\mathrm{NMe}$ at the corresponding $\mathrm{QM}$ and $\mathrm{QM} / \mathrm{MM}$ (with $\mathrm{QM}=\mathrm{B} 3 \mathrm{LYP} / 6-31+\mathrm{G}^{*}$ ) optimized geometries 
Table S1. The QEq and EEM parameters implemented in the QMMM program ${ }^{a}$

\begin{tabular}{ccccccc}
\hline Element & \multicolumn{2}{c}{$\mathrm{SCT}^{b}$} & \multicolumn{2}{c}{$\mathrm{BT}^{C}$} & \multicolumn{2}{c}{$\mathrm{EEM}^{d}$} \\
\cline { 2 - 7 } $\mathrm{A}$ & $\chi_{\mathrm{A}}^{0}(\mathrm{eV})$ & $J_{\mathrm{AA}}^{0}(\mathrm{eV})$ & $\chi_{\mathrm{A}}^{0}(\mathrm{eV})$ & $J_{\mathrm{AA}}^{0}(\mathrm{eV})$ & $\chi_{\mathrm{A}}^{0}(\mathrm{eV})$ & $\eta_{\mathrm{A}}^{*}(\mathrm{eV})$ \\
\hline $\mathrm{Li}$ & 3.006 & 4.772 & $\mathrm{n} / \mathrm{a}$ & $\mathrm{n} / \mathrm{a}$ & $\mathrm{n} / \mathrm{a}$ & $\mathrm{n} / \mathrm{a}$ \\
$\mathrm{C}$ & 5.343 & 10.126 & 5.07305 & 10.06444 & 2.30 & 9.1 \\
$\mathrm{~N}$ & 6.899 & 11.760 & 7.73699 & 12.96908 & 7.20 & 13.2 \\
$\mathrm{O}$ & 8.741 & 13.364 & 8.27885 & 14.93241 & 5.10 & 11.1 \\
$\mathrm{~F}$ & 10.874 & 14.948 & $\mathrm{n} / \mathrm{a}$ & $\mathrm{n} / \mathrm{a}$ & $\mathrm{n} / \mathrm{a}$ & $\mathrm{n} / \mathrm{a}$ \\
$\mathrm{Na}$ & 2.843 & 4.592 & $\mathrm{n} / \mathrm{a}$ & $\mathrm{n} / \mathrm{a}$ & $\mathrm{n} / \mathrm{a}$ & $\mathrm{n} / \mathrm{a}$ \\
$\mathrm{Si}$ & 4.168 & 6.974 & $\mathrm{n} / \mathrm{a}$ & $\mathrm{n} / \mathrm{a}$ & $\mathrm{n} / \mathrm{a}$ & $\mathrm{n} / \mathrm{a}$ \\
$\mathrm{P}$ & 5.463 & 8.000 & $\mathrm{n} / \mathrm{a}$ & $\mathrm{n} / \mathrm{a}$ & $\mathrm{n} / \mathrm{a}$ & $\mathrm{n} / \mathrm{a}$ \\
$\mathrm{S}$ & 6.928 & 8.972 & $\mathrm{n} / \mathrm{a}$ & $\mathrm{n} / \mathrm{a}$ & $\mathrm{n} / \mathrm{a}$ & $\mathrm{n} / \mathrm{a}$ \\
$\mathrm{Cl}$ & 8.564 & 9.892 & $\mathrm{n} / \mathrm{a}$ & $\mathrm{n} / \mathrm{a}$ & $\mathrm{n} / \mathrm{a}$ & $\mathrm{n} / \mathrm{a}$ \\
$\mathrm{K}$ & 2.421 & 3.84 & $\mathrm{n} / \mathrm{a}$ & $\mathrm{n} / \mathrm{a}$ & $\mathrm{n} / \mathrm{a}$ & $\mathrm{n} / \mathrm{a}$ \\
$\mathrm{Br}$ & 7.790 & 8.850 & $\mathrm{n} / \mathrm{a}$ & $\mathrm{n} / \mathrm{a}$ & $\mathrm{n} / \mathrm{a}$ & $\mathrm{n} / \mathrm{a}$ \\
$\mathrm{Rb}$ & 2.331 & 3.692 & $\mathrm{n} / \mathrm{a}$ & $\mathrm{n} / \mathrm{a}$ & $\mathrm{n} / \mathrm{a}$ & $\mathrm{n} / \mathrm{a}$ \\
$\mathrm{I}$ & 6.822 & 7.524 & $\mathrm{n} / \mathrm{a}$ & $\mathrm{n} / \mathrm{a}$ & $\mathrm{n} / \mathrm{a}$ & $\mathrm{n} / \mathrm{a}$ \\
$\mathrm{Cs}$ & 2.183 & 3.422 & $\mathrm{n} / \mathrm{a}$ & $\mathrm{n} / \mathrm{a}$ & $\mathrm{n} / \mathrm{a}$ & $\mathrm{n} / \mathrm{a}$ \\
$\mathrm{H}$ & 4.528 & 13.890 & 4.42211 & 13.84036 & 1.00 & 13.8 \\
\hline
\end{tabular}

a. $\quad \chi_{\mathrm{A}}^{0}$ is the electronegativity of an isolated atom. Note that the parameters tabulated here are in unit of $\mathrm{eV}$, while the equations given in the text are in atomic units.

b. QEq model with the shielded Coulomb term in Rappé, A. K.; Goddard, W. A. Journal of Physical Chemistry 1991, 95, 3358.

c. QEq model in Bakowies, D.; Thiel, W. Journal of Computational Chemistry 1996, 17, 87.

d. The 4th column of Table 1 in Bultinck, P.; Langenaeker, W.; Popelier, P. L. A.; Proft, F. D.; Geerlings, P. Journal of Physical Chemistry A 2004, 108, 10359. 
Table S2. Additional MM parameters in the TINKER parameter file format

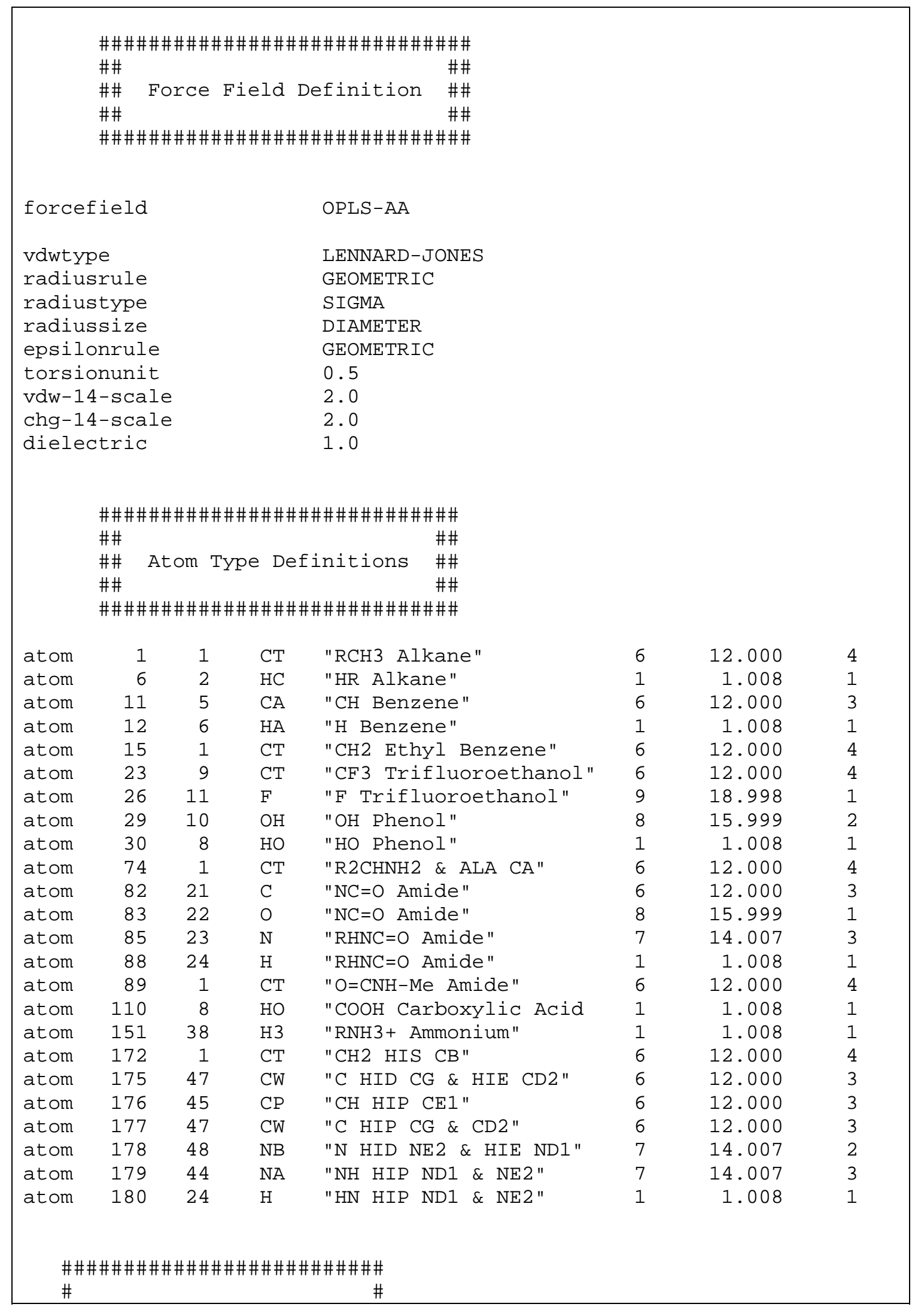




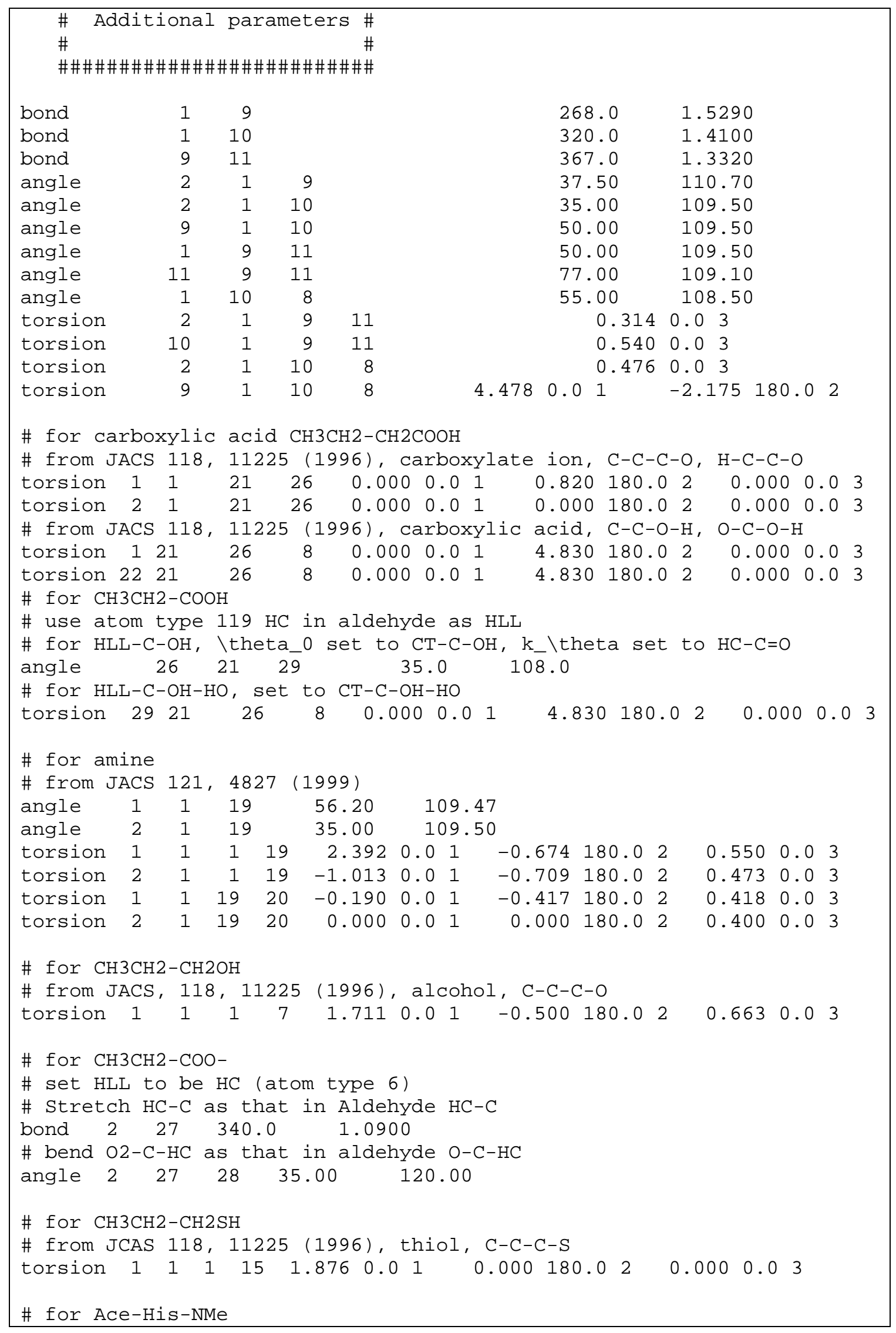




\begin{tabular}{|c|c|c|c|c|c|c|}
\hline \multicolumn{7}{|c|}{ \# for CAP: 13 HLL $27 \quad 0.713$} \\
\hline \multicolumn{7}{|c|}{ \# HLL set to the atom type of HA } \\
\hline bond & 2 & 47 & & 367.0 & $1.080 \odot$ & \\
\hline angle & 2 & 47 & 44 & $35.0 \odot$ & 121.60 & \\
\hline angle & 2 & 47 & 47 & $35 . \odot \odot$ & 130.70 & \\
\hline torsion & 24 & 44 & 47 & 2 & & $3.200180 . \odot 2$ \\
\hline torsion & 2 & 47 & 47 & 44 & & 10.750180 .02 \\
\hline torsion & 2 & 47 & 47 & 6 & & 7.250180 .02 \\
\hline torsion & 45 & 44 & 47 & 2 & & 3.200180 .02 \\
\hline \multicolumn{7}{|c|}{ \# for Ace-Tyr-NMe } \\
\hline \multicolumn{7}{|c|}{ \# for CAP ID: 13} \\
\hline \multicolumn{7}{|c|}{ \# HLL set to the atom type of HA } \\
\hline bond & 5 & 2 & & 367.0 & 1.0800 & \\
\hline angle & 5 & 5 & 2 & $35.0 \odot$ & 120.00 & \\
\hline torsion & 5 & 5 & 5 & 2 & & 7.250180 .02 \\
\hline torsion & 6 & 5 & 5 & 2 & & 7.250180 .02 \\
\hline
\end{tabular}


Table S3. Atom types, full-QM optimized coordinates (at the HF/MIDI! level), and connectivities for the amino acids in the tinker .xyz file format

A. Ace-Lys-NMe

\begin{tabular}{|c|c|c|c|c|c|c|c|c|c|}
\hline 34 & Lys & ine & & & & & & & \\
\hline 1 & CT & $-\odot .179528$ & $-\odot .673839$ & $\odot .284769$ & 1 & 2 & 4 & 5 & 6 \\
\hline 2 & C & -0.238074 & $-\odot .669464$ & 1.797939 & 82 & 1 & 3 & 7 & \\
\hline 3 & 0 & 0.481619 & -1.354202 & 2.493106 & 83 & 2 & & & \\
\hline 4 & $\mathrm{HC}$ & $\odot .645157$ & -1.302269 & $-\odot . \odot 2222 \odot$ & 6 & 1 & & & \\
\hline 5 & $\mathrm{HC}$ & $-\odot .035109$ & $\odot .330602$ & $-\odot .103686$ & 6 & 1 & & & \\
\hline 6 & $\mathrm{HC}$ & -1.101823 & -1.072856 & -0.133410 & 6 & 1 & & & \\
\hline 7 & $\mathrm{~N}$ & -1.153057 & 0.188941 & 2.339360 & 85 & 2 & 8 & 11 & \\
\hline 8 & CT & -1.367737 & $\odot .238731$ & 3.782811 & 74 & 7 & 9 & 12 & 13 \\
\hline 9 & C & -2.346913 & $-\odot .873062$ & 4.211802 & 82 & 8 & 10 & 29 & \\
\hline 10 & 0 & -3.481002 & $-\odot .613791$ & $4.5660 \odot 2$ & 83 & 9 & & & \\
\hline 11 & $\mathrm{H}$ & -1.889492 & $\odot .537339$ & 1.745895 & 88 & 7 & & & \\
\hline 12 & $\mathrm{HC}$ & $-\odot .402978$ & ๑. 062408 & 4.241086 & 6 & 8 & & & \\
\hline 13 & CT & -1.912800 & 1.620156 & 4.163541 & 2 & 8 & 14 & 18 & 19 \\
\hline 14 & CT & -1.994653 & 1.812476 & 5.687903 & 2 & 13 & 15 & 20 & 21 \\
\hline 15 & CT & -2.674204 & 3.151003 & 6.047870 & 2 & 14 & 16 & 22 & 23 \\
\hline 16 & CT & -2.830332 & 3.271016 & 7.566319 & 155 & 15 & 17 & 24 & 25 \\
\hline 17 & N3 & -3.569086 & 4.570404 & 7.926600 & 146 & 16 & 26 & 27 & 28 \\
\hline 18 & $\mathrm{HC}$ & -2.907418 & 1.723247 & 3.739447 & 6 & 13 & & & \\
\hline 19 & $\mathrm{HC}$ & -1.266919 & 2.373238 & 3.719879 & 6 & 13 & & & \\
\hline 20 & $\mathrm{HC}$ & $-\Theta .995495$ & 1.778495 & 6.118653 & 6 & 14 & & & \\
\hline 21 & $\mathrm{HC}$ & -2.577064 & 0.995343 & 6.096906 & 6 & 14 & & & \\
\hline 22 & $\mathrm{HC}$ & -3.653345 & 3.183462 & 5.573725 & 6 & 15 & & & \\
\hline 23 & $\mathrm{HC}$ & -2.084817 & 3.981664 & 5.663944 & 6 & 15 & & & \\
\hline 24 & $\mathrm{HC}$ & -1.878084 & 3.316293 & 8.078631 & 6 & 16 & & & \\
\hline 25 & $\mathrm{HC}$ & -3.425675 & 2.465883 & 7.976928 & 6 & 16 & & & \\
\hline 26 & H3 & -3.687804 & 4.673335 & 8.940157 & 151 & 17 & & & \\
\hline 27 & H3 & -4.501501 & 4.590645 & 7.499087 & 151 & 17 & & & \\
\hline 28 & H3 & -3.057483 & 5.391734 & 7.586256 & 151 & 17 & & & \\
\hline 29 & $\mathrm{~N}$ & -1.839378 & -2.113621 & 4.156879 & 85 & 9 & 30 & 31 & \\
\hline 30 & CT & -2.689361 & -3.278706 & 4.378072 & 89 & 29 & 32 & 33 & 34 \\
\hline 31 & $\mathrm{H}$ & $-\odot .922463$ & -2.228066 & 3.750267 & 88 & 29 & & & \\
\hline 32 & $\mathrm{HC}$ & -2.054425 & -4.139959 & 4.547150 & 6 & 30 & & & \\
\hline 33 & $\mathrm{HC}$ & -3.336317 & -3.475788 & 3.526734 & 6 & 30 & & & \\
\hline 34 & $\mathrm{HC}$ & -3.310625 & -3.112198 & 5.248753 & 6 & 30 & & & \\
\hline
\end{tabular}


B. Ace-His ${ }^{+}-\mathrm{NMe}$

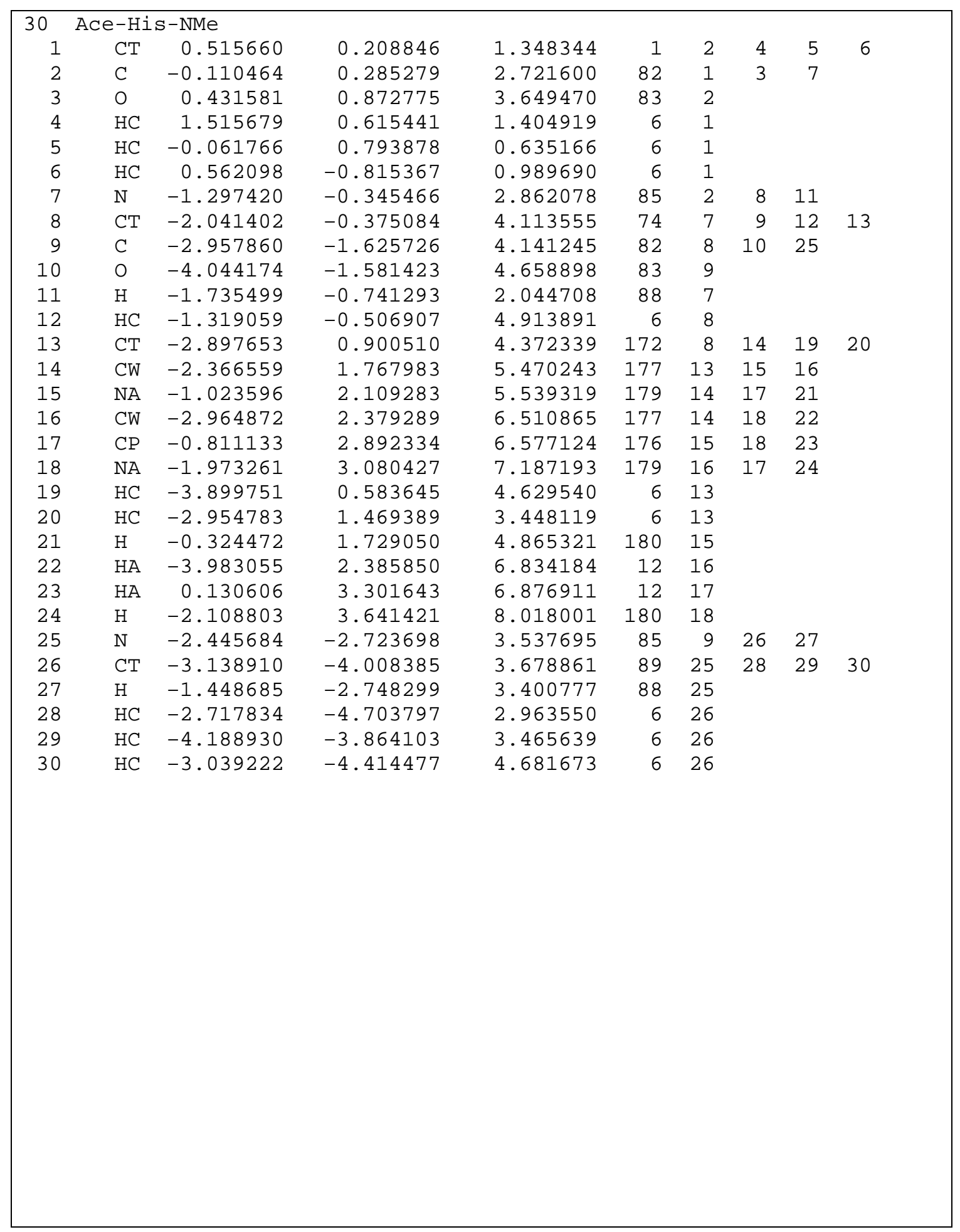


C. Ace-Glu-NMe

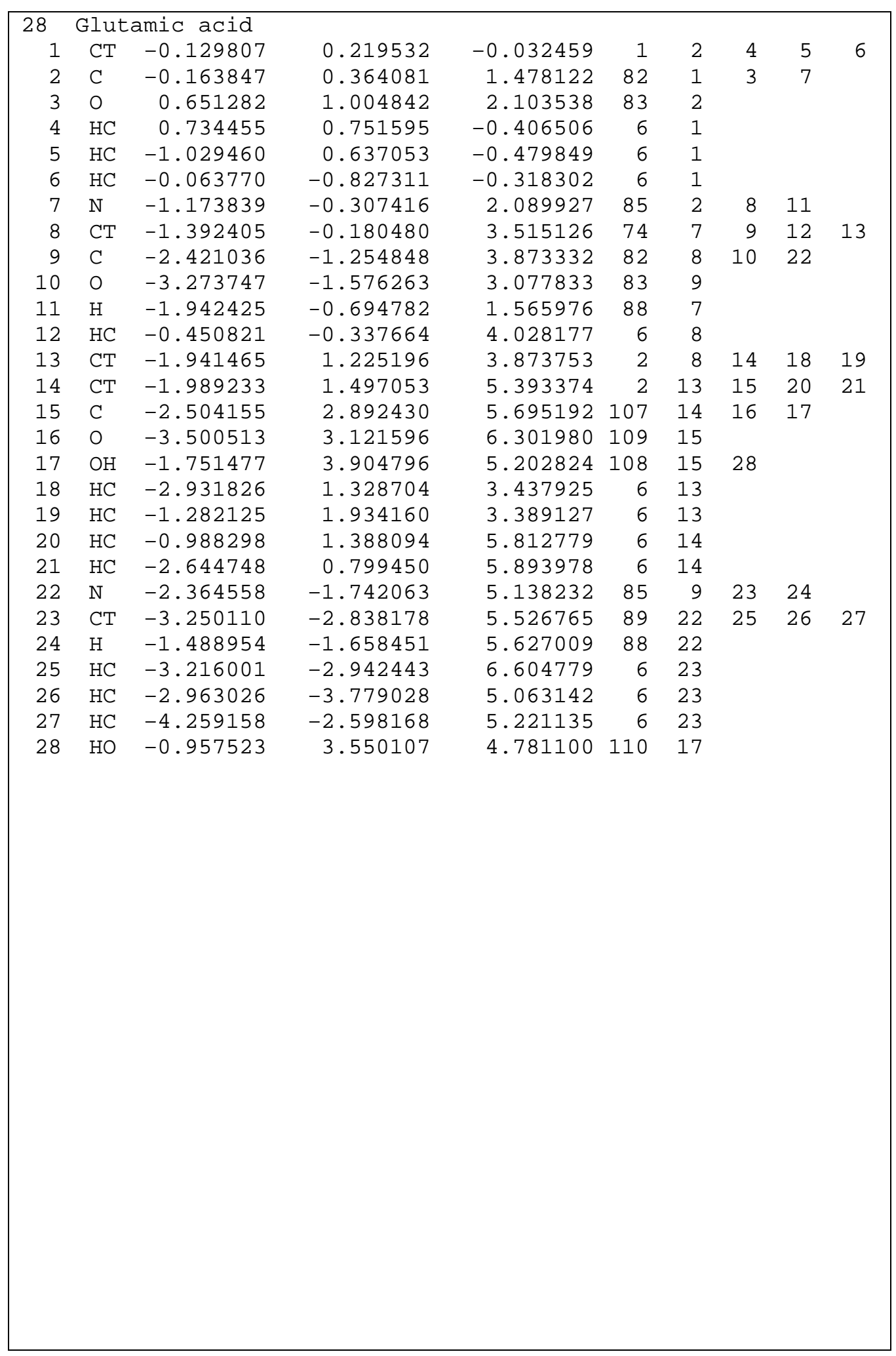


D. Ace-Tyr-NMe

\begin{tabular}{|c|c|c|c|c|c|c|c|c|c|}
\hline 33 & Ace & tyr-NMe & & & & & & & \\
\hline 1 & CT & 0.018275 & ๑. 291251 & -0.015719 & 1 & 2 & 4 & 5 & 6 \\
\hline 2 & C & -0.043351 & $\odot .390078$ & 1.499130 & 82 & 1 & 3 & 7 & \\
\hline 3 & 0 & 0.771766 & $\odot .996378$ & 2.157152 & 83 & 2 & & & \\
\hline 4 & $\mathrm{HC}$ & $\odot .89 \odot 255$ & $\odot .832853$ & $-\odot .356911$ & 6 & 1 & & & \\
\hline 5 & $\mathrm{HC}$ & $-\odot .872737$ & $\odot .722509$ & -0.467177 & 6 & 1 & & & \\
\hline 6 & $\mathrm{HC}$ & ๑. 089687 & $-\odot .746924$ & -0.331245 & 6 & 1 & & & \\
\hline 7 & $\mathrm{~N}$ & -1.079665 & -0.276058 & 2.066319 & 85 & 2 & 8 & 11 & \\
\hline 8 & CT & -1.314824 & $-\odot .224474$ & 3.491965 & 74 & 7 & 9 & 12 & 13 \\
\hline 9 & C & -2.316567 & -1.335684 & 3.798269 & 82 & 8 & 10 & 28 & \\
\hline 10 & 0 & -3.155890 & -1.647388 & 2.980829 & 83 & 9 & & & \\
\hline 11 & $\mathrm{H}$ & -1.816686 & $-\odot .685340$ & 1.515477 & 88 & 7 & & & \\
\hline 12 & $\mathrm{HC}$ & $-\odot .378849$ & $-\odot .379895$ & 4.012287 & 6 & 8 & & & \\
\hline 13 & CT & -1.914395 & 1.156133 & 3.906886 & 15 & 8 & 14 & 21 & 22 \\
\hline 14 & CA & -2.127468 & 1.293906 & 5.395246 & 11 & 13 & 15 & 16 & \\
\hline 15 & CA & -1.046833 & 1.501200 & 6.246272 & 11 & 14 & 17 & 23 & \\
\hline 16 & CA & -3.388076 & 1.210881 & 5.959144 & 11 & 14 & 18 & 24 & \\
\hline 17 & CA & -1.215215 & 1.607538 & 7.610140 & 11 & 15 & 19 & 25 & \\
\hline 18 & CA & -3.574452 & 1.319907 & 7.326718 & 11 & 16 & 19 & 26 & \\
\hline 19 & CA & -2.486171 & 1.516962 & 8.156583 & 28 & 17 & 18 & 20 & \\
\hline 20 & $\mathrm{OH}$ & -2.595661 & 1.630670 & 9.515853 & 29 & 19 & 27 & & \\
\hline 21 & $\mathrm{HC}$ & -2.849420 & 1.279999 & 3.367750 & 6 & 13 & & & \\
\hline 22 & $\mathrm{HC}$ & -1.215647 & 1.905338 & 3.551910 & 6 & 13 & & & \\
\hline 23 & HA & $-\odot .057043$ & 1.591243 & 5.828826 & 12 & 15 & & & \\
\hline 24 & HA & -4.245486 & 1.062272 & 5.323869 & 12 & 16 & & & \\
\hline 25 & HA & -0.381120 & 1.772291 & 8.267387 & 12 & 17 & & & \\
\hline 26 & HA & -4.565683 & 1.256864 & 7.744318 & 12 & 18 & & & \\
\hline 27 & $\mathrm{HO}$ & -3.532793 & 1.576813 & 9.747151 & 30 & 20 & & & \\
\hline 28 & $\mathrm{~N}$ & -2.196523 & -1.925272 & 5.010335 & 85 & 9 & 29 & 30 & \\
\hline 29 & CT & -3.251262 & -2.804800 & 5.503192 & 89 & 28 & 31 & 32 & 33 \\
\hline 30 & $\mathrm{H}$ & -1.634843 & -1.449448 & 5.696512 & 88 & 28 & & & \\
\hline 31 & $\mathrm{HC}$ & -2.839449 & -3.450343 & 6.270760 & 6 & 29 & & & \\
\hline 32 & $\mathrm{HC}$ & -3.608942 & -3.405827 & 4.679096 & 6 & 29 & & & \\
\hline 33 & $\mathrm{HC}$ & -4.087175 & -2.245480 & 5.918524 & 6 & 29 & & & \\
\hline
\end{tabular}


Table S4. Atomic charges derived from the full-QM and selected QM/MM calculations (with $\mathrm{QM}=\mathrm{HF} / \mathrm{MIDI}$ !) for the amino acids ${ }^{a}$

A. Ace-Lys-NMe

\begin{tabular}{|c|c|c|c|c|c|c|}
\hline \multicolumn{2}{|l|}{ Atom } & QM & $\mathrm{RC}$ & $\begin{array}{l}\text { PBRC } \\
(\mathrm{SCT})\end{array}$ & RCD & $\begin{array}{c}\text { PBRCD } \\
(\mathrm{SCT})\end{array}$ \\
\hline \multicolumn{7}{|c|}{ PS } \\
\hline $\mathrm{C} 13$ & & -0.152 & -0.277 & -0.278 & -0.257 & -0.253 \\
\hline $\mathrm{C} 14$ & & -0.218 & 0.032 & 0.047 & 0.040 & 0.048 \\
\hline $\mathrm{C} 15$ & & 0.022 & -0.034 & -0.062 & -0.057 & -0.074 \\
\hline $\mathrm{C} 16$ & & 0.138 & 0.058 & 0.085 & 0.077 & 0.102 \\
\hline N17 & & -0.507 & -0.423 & -0.439 & -0.425 & -0.449 \\
\hline H18 & & 0.064 & 0.101 & 0.097 & 0.106 & 0.100 \\
\hline H19 & & 0.081 & 0.089 & 0.080 & 0.094 & 0.084 \\
\hline $\mathrm{H} 20$ & & 0.084 & 0.023 & 0.020 & 0.025 & 0.023 \\
\hline $\mathrm{H} 21$ & & 0.070 & 0.053 & 0.045 & 0.055 & 0.047 \\
\hline $\mathrm{H} 22$ & & 0.049 & 0.059 & 0.061 & 0.063 & 0.063 \\
\hline $\mathrm{H} 23$ & & 0.036 & 0.048 & 0.055 & 0.052 & 0.057 \\
\hline $\mathrm{H} 24$ & & 0.077 & 0.092 & 0.087 & 0.088 & 0.084 \\
\hline $\mathrm{H} 25$ & & 0.088 & 0.097 & 0.090 & 0.093 & 0.087 \\
\hline H26 & & 0.366 & 0.352 & 0.353 & 0.351 & 0.355 \\
\hline $\mathrm{H} 27$ & & 0.350 & 0.337 & 0.340 & 0.339 & 0.342 \\
\hline $\mathrm{H} 28$ & & 0.353 & 0.334 & 0.338 & 0.335 & 0.340 \\
\hline \multicolumn{7}{|c|}{ SS } \\
\hline N7 & & -0.757 & -0.500 & -0.386 & -0.547 & -0.429 \\
\hline C9 & & 0.611 & 0.500 & 0.215 & 0.453 & 0.165 \\
\hline H12 & & 0.056 & 0.060 & 0.036 & 0.013 & -0.012 \\
\hline $\mathrm{C} 2$ & & 0.891 & 0.500 & 0.435 & 0.500 & 0.434 \\
\hline H11 & & 0.360 & 0.300 & 0.221 & 0.300 & 0.222 \\
\hline O10 & & -0.515 & -0.500 & -0.359 & -0.500 & -0.359 \\
\hline N29 & & -0.579 & -0.500 & -0.302 & -0.500 & -0.301 \\
\hline \multicolumn{2}{|c|}{ Redistributed charge $\left(q_{0}\right)$} & $\mathrm{n} / \mathrm{a}$ & 0.047 & 0.047 & 0.093 & 0.093 \\
\hline \multicolumn{2}{|c|}{ Link atom (HL) } & $\mathrm{n} / \mathrm{a}$ & 0.058 & 0.079 & 0.022 & 0.043 \\
\hline
\end{tabular}


B. Deprontonated Ace-Lys-NMe

\begin{tabular}{|c|c|c|c|c|c|}
\hline Atom & $\mathrm{QM}$ & $\mathrm{RC}$ & $\begin{array}{l}\text { PBRC } \\
(\mathrm{SCT})\end{array}$ & $\mathrm{RCD}$ & $\begin{array}{l}\text { PBRCD } \\
(\mathrm{SCT})\end{array}$ \\
\hline \multicolumn{6}{|c|}{ PS } \\
\hline $\mathrm{C} 13$ & -0.018 & -0.255 & -0.256 & -0.215 & -0.207 \\
\hline C14 & -0.252 & 0.091 & 0.090 & 0.084 & 0.067 \\
\hline C15 & 0.091 & -0.062 & -0.062 & -0.062 & -0.053 \\
\hline $\mathrm{C} 16$ & 0.322 & 0.339 & 0.344 & 0.341 & 0.325 \\
\hline N17 & -0.957 & -0.961 & -0.961 & -0.960 & -0.957 \\
\hline H1 8 & 0.028 & 0.082 & 0.081 & 0.081 & 0.078 \\
\hline H19 & 0.041 & 0.066 & 0.065 & 0.067 & 0.063 \\
\hline $\mathrm{H} 20$ & 0.054 & -0.023 & -0.024 & -0.020 & -0.016 \\
\hline $\mathrm{H} 21$ & 0.067 & 0.012 & 0.007 & 0.014 & 0.014 \\
\hline $\mathrm{H} 22$ & 0.009 & 0.036 & 0.034 & 0.037 & 0.036 \\
\hline $\mathrm{H} 23$ & 0.007 & 0.035 & 0.036 & 0.034 & 0.037 \\
\hline $\mathrm{H} 24$ & 0.005 & 0.003 & 0.003 & 0.004 & 0.007 \\
\hline $\mathrm{H} 25$ & -0.055 & -0.060 & -0.063 & -0.060 & -0.058 \\
\hline $\mathrm{H} 26$ & 0.348 & 0.348 & 0.347 & 0.347 & 0.349 \\
\hline $\mathrm{H} 27$ & 0.331 & 0.338 & 0.338 & 0.338 & 0.338 \\
\hline \multicolumn{6}{|c|}{ SS } \\
\hline N7 & -0.660 & -0.500 & -0.362 & -0.547 & -0.404 \\
\hline C9 & 0.692 & 0.500 & 0.247 & 0.453 & 0.197 \\
\hline H12 & 0.082 & 0.060 & 0.073 & 0.013 & 0.024 \\
\hline $\mathrm{C} 2$ & 0.874 & 0.500 & 0.378 & 0.500 & 0.377 \\
\hline H11 & 0.332 & 0.300 & 0.216 & 0.300 & 0.216 \\
\hline O10 & -0.520 & -0.500 & -0.347 & -0.500 & -0.347 \\
\hline $\mathrm{N} 28$ & -0.674 & -0.500 & -0.345 & -0.500 & -0.343 \\
\hline $\begin{array}{l}\text { Redistributed } \\
\text { charge }\left(q_{0}\right)\end{array}$ & $\mathrm{n} / \mathrm{a}$ & 0.047 & 0.047 & 0.093 & 0.093 \\
\hline Link atom (HL) & $\mathrm{n} / \mathrm{a}$ & 0.011 & 0.020 & -0.030 & -0.022 \\
\hline
\end{tabular}


C. Ace-His-NMe

\begin{tabular}{|c|c|c|c|c|c|}
\hline Atom & $\mathrm{QM}$ & $\mathrm{RC}$ & $\begin{array}{l}\text { PBRC } \\
(\mathrm{SCT})\end{array}$ & RCD & $\begin{array}{c}\text { PBRCD } \\
(\mathrm{SCT})\end{array}$ \\
\hline \multicolumn{6}{|c|}{ PS } \\
\hline $\mathrm{C} 13$ & -0.376 & -0.514 & -0.544 & -0.475 & -0.512 \\
\hline $\mathrm{C} 14$ & 0.162 & 0.284 & 0.314 & 0.277 & 0.309 \\
\hline N15 & -0.130 & -0.269 & -0.288 & -0.267 & -0.286 \\
\hline $\mathrm{C} 16$ & -0.207 & -0.281 & -0.277 & -0.277 & -0.275 \\
\hline $\mathrm{C} 17$ & 0.074 & 0.075 & 0.104 & 0.076 & 0.104 \\
\hline N18 & -0.241 & -0.155 & -0.187 & -0.154 & -0.185 \\
\hline H19 & 0.168 & 0.186 & 0.179 & 0.186 & 0.180 \\
\hline $\mathrm{H} 20$ & 0.151 & 0.226 & 0.219 & 0.224 & 0.218 \\
\hline $\mathrm{H} 21$ & 0.321 & 0.451 & 0.462 & 0.453 & 0.464 \\
\hline $\mathrm{H} 22$ & 0.250 & 0.268 & 0.261 & 0.269 & 0.262 \\
\hline $\mathrm{H} 23$ & 0.229 & 0.237 & 0.227 & 0.238 & 0.228 \\
\hline $\mathrm{H} 24$ & 0.385 & 0.369 & 0.372 & 0.369 & 0.373 \\
\hline \multicolumn{6}{|c|}{$\mathrm{SS}$} \\
\hline N7 & -0.582 & -0.500 & -0.409 & -0.547 & -0.451 \\
\hline C9 & 0.664 & 0.500 & 0.217 & 0.453 & 0.167 \\
\hline H12 & 0.047 & 0.060 & -0.009 & 0.013 & -0.057 \\
\hline $\mathrm{C} 2$ & 0.762 & 0.500 & 0.361 & 0.500 & 0.359 \\
\hline H11 & 0.303 & 0.300 & 0.283 & 0.300 & 0.285 \\
\hline $\mathrm{O} 10$ & -0.515 & -0.500 & -0.290 & -0.500 & -0.289 \\
\hline N25 & -0.535 & -0.500 & -0.294 & -0.500 & -0.293 \\
\hline $\begin{array}{l}\text { Redistributed } \\
\text { charge }\left(q_{0}\right)\end{array}$ & $\mathrm{n} / \mathrm{a}$ & 0.047 & 0.047 & 0.093 & 0.093 \\
\hline Link atom (HL) & $\mathrm{n} / \mathrm{a}$ & 0.122 & 0.157 & 0.082 & 0.120 \\
\hline
\end{tabular}


D. Ace-His ${ }^{\delta}-\mathrm{NMe}$ (deprotonated at the delta position)

\begin{tabular}{|c|c|c|c|c|c|}
\hline Atom & $\mathrm{QM}$ & $\mathrm{RC}$ & $\begin{array}{l}\text { PBRC } \\
(\mathrm{SCT})\end{array}$ & RCD & $\begin{array}{c}\text { PBRCD } \\
(\mathrm{SCT})\end{array}$ \\
\hline \multicolumn{6}{|c|}{ PS } \\
\hline $\mathrm{C} 13$ & -0.397 & -0.589 & -0.564 & -0.527 & -0.528 \\
\hline C14 & 0.406 & 0.486 & 0.455 & 0.460 & 0.449 \\
\hline N15 & -0.550 & -0.599 & -0.594 & -0.594 & -0.592 \\
\hline $\mathrm{C} 16$ & -0.301 & -0.336 & -0.322 & -0.324 & -0.317 \\
\hline $\mathrm{C} 17$ & 0.198 & 0.238 & 0.242 & 0.247 & 0.249 \\
\hline N18 & -0.339 & -0.339 & -0.350 & -0.346 & -0.347 \\
\hline H19 & 0.113 & 0.165 & 0.158 & 0.160 & 0.158 \\
\hline $\mathrm{H} 20$ & 0.138 & 0.198 & 0.188 & 0.193 & 0.187 \\
\hline $\mathrm{H} 21$ & 0.222 & 0.213 & 0.210 & 0.214 & 0.211 \\
\hline $\mathrm{H} 22$ & 0.116 & 0.110 & 0.110 & 0.108 & 0.106 \\
\hline $\mathrm{H} 23$ & 0.345 & 0.348 & 0.351 & 0.351 & 0.350 \\
\hline \multicolumn{6}{|c|}{ SS } \\
\hline N7 & -0.573 & -0.500 & -0.368 & -0.547 & -0.409 \\
\hline C9 & 0.655 & 0.500 & 0.229 & 0.453 & 0.180 \\
\hline H12 & 0.101 & 0.060 & 0.057 & 0.013 & 0.010 \\
\hline $\mathrm{C} 2$ & 0.804 & 0.500 & 0.374 & 0.500 & 0.373 \\
\hline H11 & 0.333 & 0.300 & 0.269 & 0.300 & 0.266 \\
\hline $\mathrm{O} 10$ & -0.535 & -0.500 & -0.353 & -0.500 & -0.353 \\
\hline N24 & -0.631 & -0.500 & -0.348 & -0.500 & -0.347 \\
\hline $\begin{array}{l}\text { Redistributed } \\
\text { charge }\left(q_{0}\right)\end{array}$ & $\mathrm{n} / \mathrm{a}$ & 0.047 & 0.047 & 0.093 & 0.093 \\
\hline Link atom (HL) & $\mathrm{n} / \mathrm{a}$ & 0.105 & 0.115 & 0.059 & 0.074 \\
\hline
\end{tabular}


E. Ace-His ${ }^{\varepsilon}-\mathrm{NMe}$ (deprotonated at the epsilon position)

\begin{tabular}{|c|c|c|c|c|c|}
\hline Atom & QM & $\mathrm{RC}$ & $\begin{array}{l}\text { PBRC } \\
(\mathrm{SCT})\end{array}$ & $\mathrm{RCD}$ & $\begin{array}{r}\text { PBRCD } \\
(\mathrm{SCT})\end{array}$ \\
\hline \multicolumn{6}{|l|}{ PS } \\
\hline $\mathrm{C} 13$ & -0.402 & -0.480 & -0.503 & -0.449 & -0.468 \\
\hline C14 & -0.040 & 0.168 & 0.169 & 0.167 & 0.166 \\
\hline N15 & -0.280 & -0.435 & -0.429 & -0.442 & -0.434 \\
\hline $\mathrm{C} 16$ & 0.091 & -0.041 & -0.043 & -0.033 & -0.034 \\
\hline $\mathrm{C} 17$ & 0.241 & 0.252 & 0.249 & 0.258 & 0.254 \\
\hline N18 & -0.564 & -0.522 & -0.524 & -0.524 & -0.526 \\
\hline H19 & 0.134 & 0.131 & 0.132 & 0.132 & 0.133 \\
\hline $\mathrm{H} 20$ & 0.155 & 0.199 & 0.203 & 0.199 & 0.201 \\
\hline $\mathrm{H} 21$ & 0.300 & 0.412 & 0.417 & 0.416 & 0.420 \\
\hline $\mathrm{H} 22$ & 0.112 & 0.134 & 0.130 & 0.132 & 0.128 \\
\hline $\mathrm{H} 23$ & 0.099 & 0.101 & 0.101 & 0.101 & 0.101 \\
\hline \multicolumn{6}{|l|}{ SS } \\
\hline N7 & -0.874 & -0.500 & -0.369 & -0.547 & -0.411 \\
\hline C9 & 0.591 & 0.500 & 0.242 & 0.453 & 0.192 \\
\hline H12 & 0.038 & 0.060 & 0.064 & 0.013 & 0.017 \\
\hline $\mathrm{C} 2$ & 0.915 & 0.500 & 0.365 & 0.500 & 0.362 \\
\hline H11 & 0.361 & 0.300 & 0.242 & 0.300 & 0.244 \\
\hline $\mathrm{O} 10$ & -0.510 & -0.500 & -0.343 & -0.500 & -0.341 \\
\hline $\mathrm{N} 24$ & -0.542 & -0.500 & 0.341 & -0.500 & -0.342 \\
\hline $\begin{array}{c}\text { Redistributed } \\
\text { charge }\left(q_{0}\right)\end{array}$ & $\mathrm{n} / \mathrm{a}$ & 0.047 & 0.047 & 0.093 & 0.093 \\
\hline Link atom (HL) & $\mathrm{n} / \mathrm{a}$ & 0.081 & 0.098 & 0.042 & 0.058 \\
\hline
\end{tabular}


F. Ace-Tyr-NMe

\begin{tabular}{|c|c|c|c|c|c|}
\hline Atom & QM & $\mathrm{RC}$ & $\begin{array}{l}\text { PBRC } \\
(\mathrm{SCT})\end{array}$ & $\mathrm{RCD}$ & $\begin{array}{r}\text { PBRCD } \\
(\mathrm{SCT})\end{array}$ \\
\hline \multicolumn{6}{|l|}{ PS } \\
\hline $\mathrm{C} 13$ & -0.245 & -0.479 & -0.516 & -0.431 & -0.468 \\
\hline C14 & 0.014 & 0.105 & 0.109 & 0.090 & 0.098 \\
\hline $\mathrm{C} 15$ & -0.157 & -0.172 & -0.167 & -0.165 & -0.165 \\
\hline C16 & -0.038 & -0.109 & -0.110 & -0.107 & -0.110 \\
\hline $\mathrm{C} 17$ & -0.360 & -0.352 & -0.360 & -0.354 & -0.357 \\
\hline $\mathrm{C} 18$ & -0.488 & -0.457 & -0.466 & -0.457 & -0.464 \\
\hline C19 & 0.522 & 0.500 & 0.508 & 0.504 & 0.509 \\
\hline $\mathrm{O} 20$ & -0.587 & -0.587 & -0.590 & -0.586 & -0.589 \\
\hline $\mathrm{H} 21$ & 0.094 & 0.150 & 0.158 & 0.147 & 0.154 \\
\hline $\mathrm{H} 22$ & 0.129 & 0.202 & 0.212 & 0.199 & 0.208 \\
\hline $\mathrm{H} 23$ & 0.180 & 0.170 & 0.171 & 0.171 & 0.173 \\
\hline $\mathrm{H} 24$ & 0.147 & 0.154 & 0.154 & 0.156 & 0.156 \\
\hline $\mathrm{H} 25$ & 0.201 & 0.204 & 0.207 & 0.205 & 0.206 \\
\hline $\mathrm{H} 26$ & 0.191 & 0.191 & 0.192 & 0.192 & 0.192 \\
\hline $\mathrm{H} 27$ & 0.408 & 0.409 & 0.410 & 0.409 & 0.410 \\
\hline \multicolumn{6}{|l|}{ SS } \\
\hline N7 & -0.681 & -0.500 & -0.373 & -0.547 & -0.415 \\
\hline C9 & 0.735 & 0.500 & 0.236 & 0.453 & 0.186 \\
\hline H12 & 0.073 & 0.060 & 0.063 & 0.013 & 0.015 \\
\hline $\mathrm{C} 2$ & 0.841 & 0.500 & 0.400 & 0.500 & 0.397 \\
\hline H11 & 0.342 & 0.300 & 0.231 & 0.300 & 0.234 \\
\hline O10 & -0.540 & -0.500 & -0.358 & -0.500 & -0.356 \\
\hline $\mathrm{N} 28$ & -0.666 & -0.500 & -0.340 & -0.500 & -0.341 \\
\hline $\begin{array}{l}\text { Redistributed } \\
\text { charge }\left(q_{0}\right)\end{array}$ & $\mathrm{n} / \mathrm{a}$ & 0.047 & 0.047 & 0.093 & 0.093 \\
\hline Link atom (HL) & $\mathrm{n} / \mathrm{a}$ & 0.070 & 0.089 & 0.027 & 0.047 \\
\hline
\end{tabular}


G. Deprotonated Ace-Tyr-NMe

\begin{tabular}{|c|c|c|c|c|c|}
\hline Atom & QM & $\mathrm{RC}$ & $\begin{array}{l}\text { PBRC } \\
(\mathrm{SCT})\end{array}$ & RCD & $\begin{array}{r}\text { PBRCD } \\
(\mathrm{SCT})\end{array}$ \\
\hline \multicolumn{6}{|l|}{ PS } \\
\hline $\mathrm{C} 13$ & -0.208 & -0.315 & -0.362 & -0.295 & -0.314 \\
\hline $\mathrm{C} 14$ & -0.200 & -0.155 & -0.168 & -0.168 & -0.186 \\
\hline $\mathrm{C} 15$ & -0.088 & -0.084 & -0.064 & -0.066 & -0.060 \\
\hline $\mathrm{C} 16$ & -0.012 & -0.080 & -0.081 & -0.066 & -0.074 \\
\hline $\mathrm{C} 17$ & -0.545 & -0.547 & -0.574 & -0.557 & -0.567 \\
\hline $\mathrm{C} 18$ & -0.569 & -0.570 & -0.584 & -0.572 & -0.589 \\
\hline C19 & 0.753 & 0.739 & 0.748 & 0.743 & 0.756 \\
\hline $\mathrm{O} 20$ & -0.730 & -0.737 & -0.732 & -0.735 & -0.733 \\
\hline $\mathrm{H} 21$ & 0.076 & 0.093 & 0.112 & 0.099 & 0.111 \\
\hline $\mathrm{H} 22$ & 0.104 & 0.142 & 0.168 & 0.146 & 0.166 \\
\hline $\mathrm{H} 23$ & 0.144 & 0.124 & 0.120 & 0.122 & 0.121 \\
\hline $\mathrm{H} 24$ & 0.107 & 0.117 & 0.121 & 0.112 & 0.121 \\
\hline $\mathrm{H} 25$ & 0.136 & 0.143 & 0.153 & 0.144 & 0.150 \\
\hline $\mathrm{H} 26$ & 0.134 & 0.145 & 0.151 & 0.144 & 0.153 \\
\hline \multicolumn{6}{|l|}{$\mathrm{SS}$} \\
\hline N7 & -0.655 & -0.500 & -0.346 & -0.547 & -0.387 \\
\hline C9 & 0.790 & 0.500 & 0.264 & 0.453 & 0.215 \\
\hline $\mathrm{H} 12$ & 0.059 & 0.060 & 0.108 & 0.013 & 0.061 \\
\hline $\mathrm{C} 2$ & 0.800 & 0.500 & 0.323 & 0.500 & 0.320 \\
\hline H1 1 & 0.321 & 0.300 & 0.189 & 0.300 & 0.191 \\
\hline $\mathrm{O} 10$ & -0.577 & -0.500 & -0.403 & -0.500 & -0.402 \\
\hline N27 & -0.686 & -0.500 & -0.275 & -0.500 & -0.278 \\
\hline $\begin{array}{l}\text { Redistributed } \\
\text { charge }\left(q_{0}\right)\end{array}$ & $\mathrm{n} / \mathrm{a}$ & 0.047 & 0.047 & 0.093 & 0.093 \\
\hline Link atom (HL) & $\mathrm{n} / \mathrm{a}$ & -0.013 & -0.008 & -0.053 & -0.054 \\
\hline
\end{tabular}


H. Ace-Glu-NMe

\begin{tabular}{|c|c|c|c|c|c|}
\hline Atom & QM & $\mathrm{RC}$ & $\begin{array}{l}\text { PBRC } \\
(\mathrm{SCT})\end{array}$ & RCD & $\begin{array}{r}\text { PBRCD } \\
(\mathrm{SCT})\end{array}$ \\
\hline \multicolumn{6}{|l|}{ PS } \\
\hline $\mathrm{C} 13$ & 0.148 & -0.090 & -0.083 & -0.056 & -0.054 \\
\hline C14 & -0.439 & -0.212 & -0.193 & -0.215 & -0.191 \\
\hline $\mathrm{C} 15$ & 0.788 & 0.711 & 0.709 & 0.705 & 0.702 \\
\hline O16 & -0.493 & -0.483 & -0.487 & -0.479 & -0.483 \\
\hline $\mathrm{C} 17$ & -0.630 & -0.596 & -0.595 & -0.593 & -0.593 \\
\hline $\mathrm{H} 18$ & 0.045 & 0.077 & 0.069 & 0.077 & 0.070 \\
\hline H19 & 0.002 & 0.065 & 0.054 & 0.066 & 0.056 \\
\hline $\mathrm{H} 20$ & 0.105 & 0.050 & 0.044 & 0.055 & 0.047 \\
\hline $\mathrm{H} 21$ & 0.078 & 0.058 & 0.048 & 0.060 & 0.049 \\
\hline $\mathrm{H} 28$ & 0.438 & 0.425 & 0.425 & 0.424 & 0.425 \\
\hline \multicolumn{6}{|l|}{ SS } \\
\hline N7 & -0.714 & -0.500 & -0.374 & -0.547 & -0.416 \\
\hline C9 & 0.676 & 0.500 & 0.232 & 0.453 & 0.181 \\
\hline H12 & 0.127 & 0.060 & 0.046 & 0.013 & -0.003 \\
\hline $\mathrm{C} 2$ & 0.847 & 0.500 & 0.413 & 0.500 & 0.411 \\
\hline H11 & 0.365 & 0.300 & 0.243 & 0.300 & 0.245 \\
\hline $\mathrm{O} 10$ & -0.525 & -0.500 & -0.341 & -0.500 & -0.339 \\
\hline $\mathrm{N} 22$ & -0.408 & -0.500 & -0.359 & -0.500 & -0.359 \\
\hline $\begin{array}{c}\text { Redistributed } \\
\text { charge }\left(q_{0}\right)\end{array}$ & $\mathrm{n} / \mathrm{a}$ & 0.047 & 0.047 & 0.093 & 0.093 \\
\hline Link atom (HL) & $\mathrm{n} / \mathrm{a}$ & -0.005 & 0.009 & -0.045 & -0.028 \\
\hline
\end{tabular}


I. Deprontonated AceE-Glu-NMe

\begin{tabular}{|c|c|c|c|c|c|}
\hline Atom & QM & $\mathrm{RC}$ & $\begin{array}{l}\text { PBRC } \\
(\mathrm{SCT})\end{array}$ & RCD & $\begin{array}{r}\text { PBRCD } \\
(\mathrm{SCT})\end{array}$ \\
\hline \multicolumn{6}{|l|}{ PS } \\
\hline $\mathrm{C} 13$ & 0.039 & -0.314 & -0.315 & -0.265 & -0.264 \\
\hline $\mathrm{C} 14$ & -0.208 & -0.017 & -0.024 & -0.038 & -0.037 \\
\hline $\mathrm{C} 15$ & 0.726 & 0.788 & 0.793 & 0.790 & 0.790 \\
\hline O16 & -0.742 & -0.776 & -0.774 & -0.773 & -0.770 \\
\hline $\mathrm{C} 17$ & -0.730 & -0.758 & -0.755 & -0.758 & -0.754 \\
\hline H18 & -0.004 & 0.075 & 0.085 & 0.074 & 0.083 \\
\hline H19 & 0.042 & 0.113 & 0.115 & 0.112 & 0.112 \\
\hline $\mathrm{H} 20$ & 0.013 & -0.033 & -0.034 & -0.025 & -0.028 \\
\hline $\mathrm{H} 21$ & 0.023 & -0.050 & -0.055 & -0.043 & -0.050 \\
\hline \multicolumn{6}{|l|}{ SS } \\
\hline N7 & -0.785 & -0.500 & -0.319 & -0.547 & -0.360 \\
\hline C9 & 0.668 & 0.500 & 0.282 & 0.453 & 0.232 \\
\hline H12 & 0.049 & 0.060 & 0.119 & 0.013 & 0.071 \\
\hline $\mathrm{C} 2$ & 0.909 & 0.500 & 0.326 & 0.500 & 0.325 \\
\hline H11 & 0.331 & 0.300 & 0.206 & 0.300 & 0.206 \\
\hline $\mathrm{O} 10$ & -0.551 & -0.500 & -0.391 & -0.500 & -0.389 \\
\hline $\mathrm{N} 22$ & -0.634 & -0.500 & -0.363 & -0.500 & -0.366 \\
\hline $\begin{array}{l}\text { Redistributed } \\
\text { charge }\left(q_{0}\right)\end{array}$ & $\mathrm{n} / \mathrm{a}$ & 0.047 & 0.047 & 0.093 & 0.093 \\
\hline Link atom (HL) & $\mathrm{n} / \mathrm{a}$ & -0.029 & -0.036 & -0.074 & -0.082 \\
\hline
\end{tabular}

a. The geometries were optimized at the given QM and QM/MM levels. Only the QEq-SCT results are shown for the PBRC and PBRCD calculations. The side chain is the PS, and the backbone is the SS. The QM level is HF/MIDI!, and the MM force field is OPLS-AA. For the PS atoms, the QM ESP charges are listed. For the SS atoms, the QM ESP charges are given for the full-QM calculations, the OPLS-AA charges for the RC and RCD calculations, and the QEq charges for the PBRC and PBRCD calculations. The redistributed charge $q_{0}$ was fixed to $q_{\mathrm{M} 1} / 3$ in the RC and PBRC calculations and to 
$2 q_{\mathrm{M} 1} / 3$ in the RCD and PBRCD calculations, where $q_{\mathrm{M} 1}$ is the OPLS-AA charge on the M1 atom in the SS. The QM ESP charge is listed for the link atom, which is part of the CPS. 
Table S5. The convergence of the atomic charges of the heavy atoms in the PS and SS and the convergence of embedded-QM (with QM = HF/MIDI!) energies for the CPS in the PBRC calculations employing the QEq-SCT model for the Ace-His ${ }^{+}-\mathrm{NMe}$ system and its deprotonated forms $^{a}$
A. Ace-His ${ }^{+}-\mathrm{NMe}$

\begin{tabular}{crrr}
\hline Atom & Iteration 1 & Iteration 2 & Iteration 3 \\
\hline $\mathrm{C} 13$ & -0.542 & -0.544 & -0.544 \\
$\mathrm{C} 14$ & 0.312 & 0.314 & 0.314 \\
$\mathrm{~N} 15$ & -0.287 & -0.288 & -0.288 \\
$\mathrm{C} 16$ & -0.276 & -0.277 & -0.277 \\
$\mathrm{C} 17$ & 0.104 & 0.104 & 0.104 \\
$\mathrm{~N} 18$ & -0.187 & -0.187 & -0.187 \\
& & & \\
$\mathrm{~N} 7$ & -0.403 & -0.408 & -0.409 \\
$\mathrm{C} 9$ & 0.223 & 0.218 & 0.217 \\
$\mathrm{C} 2$ & 0.357 & 0.361 & 0.361 \\
$\mathrm{O} 10$ & -0.295 & -0.291 & -0.290 \\
$\mathrm{~N} 25$ & -0.301 & -0.295 & -0.294 \\
& & & -263.00106 \\
$E_{\mathrm{QM}}\left(\mathrm{E}_{\mathrm{h}}\right)$ & -262.99939 & -263.00079 & \\
\hline
\end{tabular}


B. Ace-His ${ }^{\delta}-\mathrm{NMe}$ (deprotonated at the delta position)

\begin{tabular}{crrr}
\hline Atom & Iteration 1 & Iteration 2 & Iteration 3 \\
\hline $\mathrm{C} 13$ & -0.563 & -0.564 & -0.564 \\
$\mathrm{C} 14$ & 0.455 & 0.455 & 0.455 \\
$\mathrm{~N} 15$ & -0.593 & -0.594 & -0.594 \\
$\mathrm{C} 16$ & -0.322 & -0.322 & -0.322 \\
$\mathrm{C} 17$ & 0.242 & 0.242 & 0.242 \\
$\mathrm{~N} 18$ & -0.349 & -0.350 & -0.350 \\
& & & \\
$\mathrm{~N} 7$ & -0.366 & -0.368 & -0.368 \\
$\mathrm{C} 9$ & 0.234 & 0.229 & 0.229 \\
$\mathrm{C} 2$ & 0.372 & 0.374 & 0.374 \\
$\mathrm{O} 10$ & -0.356 & -0.354 & -0.353 \\
$\mathrm{~N} 24$ & -0.350 & -0.348 & -0.348 \\
& & & \\
$E_{\mathrm{QM}}\left(\mathrm{E}_{\mathrm{h}}\right)$ & -262.60145 & -262.60169 & -262.60180 \\
\hline
\end{tabular}


C. Ace-His ${ }^{\varepsilon}$-NMe (deprotonated at the epsilon position) ${ }^{a}$

\begin{tabular}{crrr}
\hline Atom & Iteration 1 & Iteration 2 & Iteration 3 \\
\hline $\mathrm{C} 13$ & -0.502 & -0.503 & -0.503 \\
$\mathrm{C} 14$ & 0.169 & 0.169 & 0.170 \\
$\mathrm{~N} 15$ & -0.429 & -0.429 & -0.429 \\
$\mathrm{C} 16$ & -0.043 & -0.043 & -0.043 \\
$\mathrm{C} 17$ & 0.249 & 0.249 & 0.249 \\
$\mathrm{~N} 18$ & -0.524 & -0.524 & -0.524 \\
& & & \\
$\mathrm{~N} 7$ & -0.367 & -0.369 & -0.369 \\
$\mathrm{C} 9$ & 0.245 & 0.242 & 0.242 \\
$\mathrm{C} 2$ & 0.364 & 0.365 & 0.365 \\
$\mathrm{O} 10$ & -0.345 & -0.343 & -0.343 \\
$\mathrm{~N} 24$ & -0.345 & -0.342 & -0.341 \\
& & & -262.59952 \\
$E_{\mathrm{QM}}\left(\mathrm{E}_{\mathrm{h}}\right)$ & -262.59976 & -262.59950 & \\
\hline
\end{tabular}

a. Geometries optimized with QEq parameters with SCT of Rappé and Goddard. 
Table S6. Comparison of the charges on H11, H21 and N15, the distance between H11 and H21, and the distance between $\mathrm{H} 11$ and N15 for the Ace-His ${ }^{+}-\mathrm{NMe}$ system and its deprotonated forms (with QM $=\mathrm{HF} / \mathrm{MIDI})^{a}$

\begin{tabular}{|c|c|c|c|c|}
\hline & & Ace-His ${ }^{+}-\mathrm{NMe}$ & Ace-His ${ }^{\delta}-\mathrm{NMe}$ & Ace-His ${ }^{\varepsilon}-\mathrm{NMe}$ \\
\hline \multicolumn{5}{|l|}{ QM } \\
\hline & $Q_{\mathrm{H} 11}^{b}$ & 0.303 & 0.333 & 0.361 \\
\hline & $Q_{\mathrm{N} 15}^{b}$ & -0.130 & -0.550 & -0.280 \\
\hline & $Q_{\mathrm{H} 21}^{b}$ & 0.321 & $\mathrm{n} / \mathrm{a}$ & 0.300 \\
\hline & $R_{\mathrm{H} 21-\mathrm{H} 11}$ & 4.006 & $\mathrm{n} / \mathrm{a}$ & 4.145 \\
\hline & $R_{\mathrm{H} 11-\mathrm{N} 15}$ & 4.566 & 4.947 & 4.585 \\
\hline \multicolumn{5}{|l|}{$\mathrm{RC}$} \\
\hline & $Q_{\mathrm{H} 11}^{c}$ & 0.300 & 0.300 & 0.300 \\
\hline & $Q_{\mathrm{N} 15}^{b}$ & -0.269 & -0.599 & -0.435 \\
\hline & $Q_{\mathrm{H} 21}^{b}$ & 0.451 & $\mathrm{n} / \mathrm{a}$ & 0.412 \\
\hline & $R_{\mathrm{H} 21-\mathrm{H} 11}$ & 3.964 & $\mathrm{n} / \mathrm{a}$ & 4.198 \\
\hline & $R_{\mathrm{H} 11-\mathrm{N} 15}$ & 4.507 & 2.451 & 4.671 \\
\hline \multicolumn{5}{|l|}{ PBRC } \\
\hline \multicolumn{5}{|c|}{$\mathrm{SCT}$} \\
\hline & $Q_{\mathrm{H} 11}^{d}$ & 0.283 & 0.269 & 0.242 \\
\hline & $Q_{\mathrm{N} 15}^{b}$ & -0.288 & -0.594 & -0.429 \\
\hline & $Q_{\mathrm{H} 21}^{b}$ & 0.462 & $\mathrm{n} / \mathrm{a}$ & 0.417 \\
\hline & $R_{\mathrm{H} 21-\mathrm{H} 11}$ & 3.856 & $\mathrm{n} / \mathrm{a}$ & 4.162 \\
\hline & $R_{\mathrm{H} 11-\mathrm{N} 15}$ & 4.416 & 2.500 & 4.650 \\
\hline \multicolumn{5}{|c|}{ BT } \\
\hline & $Q_{\mathrm{H} 11}{ }^{c}$ & 0.526 & 0.665 & 0.469 \\
\hline & $Q_{\mathrm{N} 15}^{b}$ & -0.279 & -0.776 & -0.454 \\
\hline & $Q_{\mathrm{H} 21}^{b}$ & 0.452 & $\mathrm{n} / \mathrm{a}$ & 0.415 \\
\hline & $R_{\mathrm{H} 21-\mathrm{H} 11}$ & 3.963 & $\mathrm{n} / \mathrm{a}$ & 4.190 \\
\hline & $R_{\mathrm{H} 11-\mathrm{N} 15}$ & 4.513 & 1.659 & 4.662 \\
\hline
\end{tabular}




\begin{tabular}{|c|c|c|c|c|}
\hline \multicolumn{5}{|c|}{ EEM } \\
\hline & $Q_{\mathrm{H} 11} f$ & 0.231 & 0.221 & 0.214 \\
\hline & $Q_{\mathrm{N} 15}^{b}$ & -0.274 & -0.583 & -0.437 \\
\hline & $Q_{\mathrm{H} 21}^{b}$ & 0.454 & $\mathrm{n} / \mathrm{a}$ & 0.413 \\
\hline & $R_{\mathrm{H} 21-\mathrm{H} 11}$ & 3.912 & $\mathrm{n} / \mathrm{a}$ & 4.171 \\
\hline & $R_{\mathrm{H} 11-\mathrm{N} 15}$ & 4.465 & 2.696 & 4.654 \\
\hline \multicolumn{5}{|c|}{$\mathrm{RCD}$} \\
\hline & $Q_{\mathrm{H} 11}{ }^{c}$ & 0.300 & 0.300 & 0.300 \\
\hline & $Q_{\mathrm{N} 15}^{b}$ & -0.267 & -0.594 & -0.442 \\
\hline & $Q_{\mathrm{H} 21}^{b}$ & 0.453 & $\mathrm{n} / \mathrm{a}$ & 0.416 \\
\hline & $R_{\mathrm{H} 21-\mathrm{H} 11}$ & 3.960 & $\mathrm{n} / \mathrm{a}$ & 4.191 \\
\hline & $R_{\mathrm{H} 11-\mathrm{N} 15}$ & 4.504 & 2.481 & 4.666 \\
\hline \multicolumn{5}{|c|}{ PBRCD } \\
\hline \multicolumn{5}{|c|}{$\mathrm{SCT}$} \\
\hline & $Q_{\mathrm{H} 11}^{d}$ & 0.285 & 0.266 & 0.244 \\
\hline & $Q_{\mathrm{N} 15}^{b}$ & -0.286 & -0.592 & -0.434 \\
\hline & $Q_{\mathrm{H} 21}$ & 0.464 & $\mathrm{n} / \mathrm{a}$ & 0.420 \\
\hline & $R_{\mathrm{H} 21-\mathrm{H} 11}$ & 3.854 & $\mathrm{n} / \mathrm{a}$ & 4.154 \\
\hline & $R_{\mathrm{H} 11-\mathrm{N} 15}$ & 4.416 & 2.535 & 4.644 \\
\hline \multicolumn{5}{|c|}{ BT } \\
\hline & $Q_{\mathrm{H} 11}^{e}$ & 0.521 & 0.605 & 0.465 \\
\hline & $Q_{\mathrm{N} 15}^{b}$ & -0.277 & -0.723 & -0.450 \\
\hline & $Q_{\mathrm{H} 21}^{b}$ & 0.454 & $\mathrm{n} / \mathrm{a}$ & 0.416 \\
\hline & $R_{\mathrm{H} 21-\mathrm{H} 11}$ & 3.961 & $\mathrm{n} / \mathrm{a}$ & 4.182 \\
\hline & $R_{\mathrm{H} 11-\mathrm{N} 15}$ & 4.512 & 1.761 & 4.657 \\
\hline \multicolumn{5}{|c|}{ EEM } \\
\hline & $Q_{\mathrm{H} 11} f$ & 0.232 & 0.207 & 0.215 \\
\hline & $Q_{\mathrm{N} 15}^{b}$ & -0.272 & -0.564 & -0.443 \\
\hline & $Q_{\mathrm{H} 21}^{b}$ & 0.455 & $\mathrm{n} / \mathrm{a}$ & 0.418 \\
\hline & $R_{\mathrm{H} 21-\mathrm{H} 11}$ & 3.913 & $\mathrm{n} / \mathrm{a}$ & 4.165 \\
\hline & $R_{\mathrm{H} 11-\mathrm{N} 15}$ & 4.466 & 4.102 & 4.649 \\
\hline
\end{tabular}


a. The geometries were optimized at the given level of theory. Charges are in units of e, and distances in units of $\AA$.

b. QM ESP charges.

c. OPLS-AA charges.

d. QEq-SCT charges.

e. QEq-BT charges.

f. EEM charges. 
Table S7. Atomic charges (in the unit of e) derived from the full-QM and selected QM/MM calculations for the amino acids at the full-QM optimized geometries at the HF/MIDI! level ${ }^{a}$ A. Ace-Lys-NMe

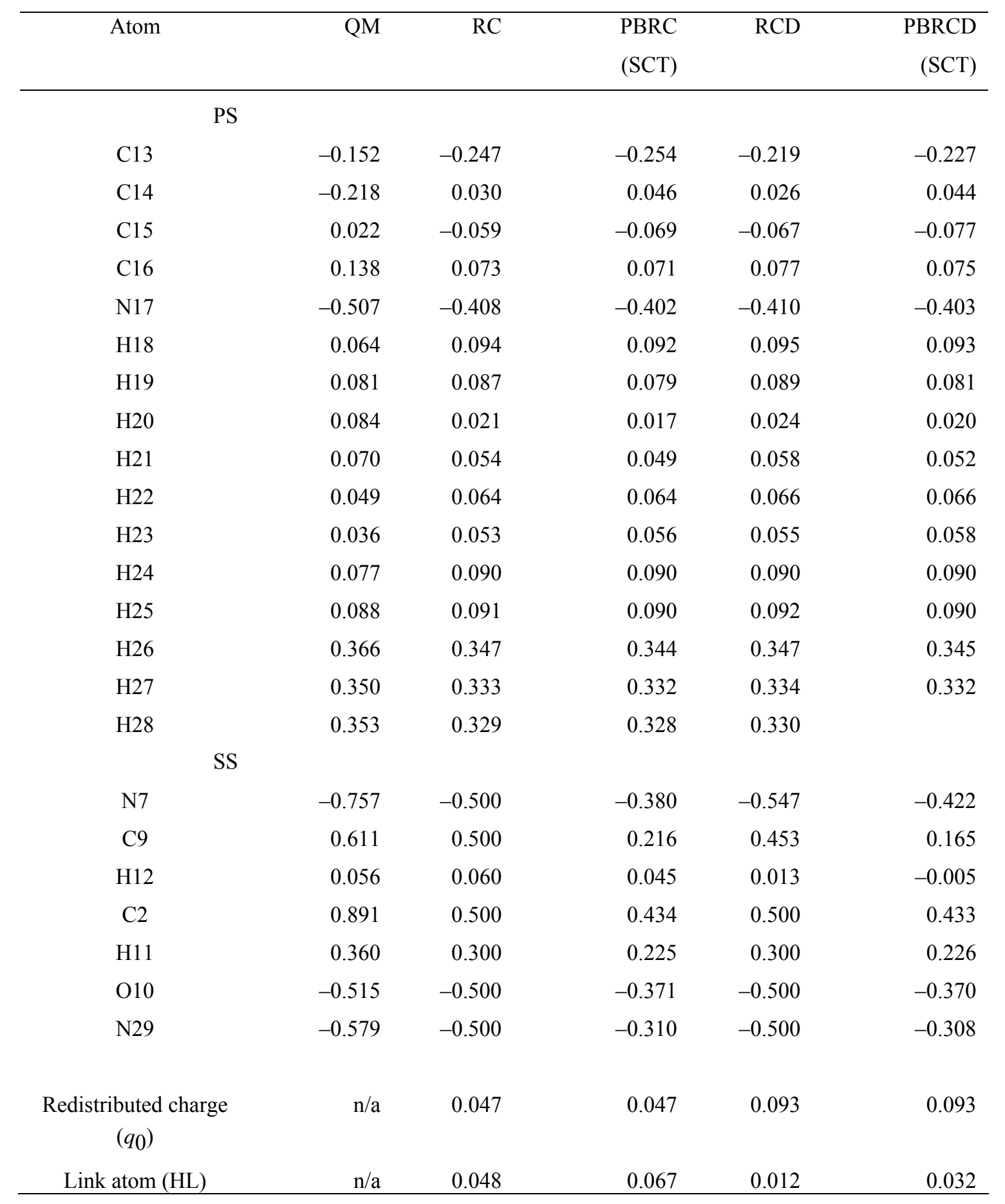


B. Deprotonated Ace-Lys-NMe

\begin{tabular}{|c|c|c|c|c|c|}
\hline Atom & $\mathrm{QM}$ & $\mathrm{RC}$ & $\begin{array}{l}\text { PBRC } \\
(\mathrm{SCT})\end{array}$ & $\mathrm{RCD}$ & $\begin{array}{r}\text { PBRCD } \\
(\mathrm{SCT})\end{array}$ \\
\hline \multicolumn{6}{|c|}{ PS } \\
\hline $\mathrm{C} 13$ & -0.018 & -0.222 & -0.227 & -0.192 & -0.198 \\
\hline C14 & -0.252 & 0.075 & 0.081 & 0.071 & 0.079 \\
\hline C15 & 0.091 & -0.054 & -0.061 & -0.062 & -0.070 \\
\hline $\mathrm{C} 16$ & 0.322 & 0.324 & 0.325 & 0.326 & 0.328 \\
\hline N17 & -0.957 & -0.956 & -0.956 & -0.955 & -0.955 \\
\hline H18 & 0.028 & 0.073 & 0.073 & 0.074 & 0.074 \\
\hline H19 & 0.041 & 0.061 & 0.059 & 0.063 & 0.061 \\
\hline $\mathrm{H} 20$ & 0.054 & -0.022 & -0.024 & -0.018 & -0.020 \\
\hline $\mathrm{H} 21$ & 0.067 & 0.013 & 0.010 & 0.017 & 0.013 \\
\hline $\mathrm{H} 22$ & 0.009 & 0.036 & 0.036 & 0.038 & 0.038 \\
\hline $\mathrm{H} 23$ & 0.007 & 0.036 & 0.039 & 0.038 & 0.041 \\
\hline $\mathrm{H} 24$ & 0.005 & 0.005 & 0.005 & 0.005 & 0.005 \\
\hline $\mathrm{H} 25$ & -0.055 & -0.058 & -0.060 & -0.057 & -0.059 \\
\hline $\mathrm{H} 26$ & 0.348 & 0.350 & 0.350 & 0.350 & 0.350 \\
\hline $\mathrm{H} 27$ & 0.331 & 0.337 & 0.337 & 0.337 & 0.337 \\
\hline \multicolumn{6}{|c|}{ SS } \\
\hline N7 & -0.660 & -0.500 & -0.359 & -0.547 & -0.401 \\
\hline C9 & 0.692 & 0.500 & 0.250 & 0.453 & 0.200 \\
\hline H12 & 0.082 & 0.060 & 0.076 & 0.013 & 0.027 \\
\hline $\mathrm{C} 2$ & 0.874 & 0.500 & 0.376 & 0.500 & 0.376 \\
\hline H11 & 0.332 & 0.300 & 0.216 & 0.300 & 0.216 \\
\hline $\mathrm{O} 10$ & -0.520 & -0.500 & -0.353 & -0.500 & -0.352 \\
\hline $\mathrm{N} 28$ & -0.674 & -0.500 & -0.347 & -0.500 & -0.346 \\
\hline $\begin{array}{l}\text { Redistributed } \\
\text { charge }\left(q_{0}\right)\end{array}$ & $\mathrm{n} / \mathrm{a}$ & 0.047 & 0.047 & 0.093 & 0.093 \\
\hline Link atom (HL) & $\mathrm{n} / \mathrm{a}$ & 0.003 & 0.012 & -0.034 & -0.025 \\
\hline
\end{tabular}


C. $A c e-\mathrm{His}^{+}-\mathrm{NMe}$

\begin{tabular}{|c|c|c|c|c|c|c|c|c|c|c|}
\hline \multicolumn{2}{|l|}{ Atom } & \multirow[t]{2}{*}{$\mathrm{QM}$} & \multirow[t]{2}{*}{$\mathrm{RC}$} & \multicolumn{3}{|c|}{ PBRC } & \multirow[t]{2}{*}{$\mathrm{RCD}$} & \multirow[b]{2}{*}{$(\mathrm{SCT})$} & \multicolumn{2}{|l|}{ PBRCD } \\
\hline & & & & $(\mathrm{SCT})$ & (BT) & (EEM) & & & (BT) & (EEM) \\
\hline \multicolumn{11}{|c|}{ PS } \\
\hline $\mathrm{C} 13$ & & -0.376 & -0.510 & -0.527 & -0.546 & -0.532 & -0.477 & -0.496 & -0.514 & -0.504 \\
\hline $\mathrm{C} 14$ & & 0.162 & 0.296 & 0.313 & 0.314 & 0.306 & 0.292 & 0.311 & 0.309 & 0.304 \\
\hline $\mathrm{N} 15$ & & -0.130 & -0.303 & -0.300 & -0.309 & -0.300 & -0.301 & -0.298 & -0.307 & -0.298 \\
\hline $\mathrm{C} 16$ & & -0.207 & -0.239 & -0.252 & -0.252 & -0.248 & -0.239 & -0.253 & -0.251 & -0.248 \\
\hline $\mathrm{C} 17$ & & 0.074 & 0.124 & 0.119 & 0.121 & 0.122 & 0.124 & 0.119 & 0.122 & 0.122 \\
\hline N18 & & -0.241 & -0.210 & -0.210 & -0.206 & -0.212 & -0.206 & -0.206 & -0.203 & -0.209 \\
\hline H19 & & 0.168 & 0.224 & 0.208 & 0.204 & 0.199 & 0.224 & 0.209 & 0.206 & 0.200 \\
\hline $\mathrm{H} 20$ & & 0.151 & 0.196 & 0.193 & 0.206 & 0.200 & 0.195 & 0.192 & 0.206 & 0.198 \\
\hline $\mathrm{H} 21$ & & 0.321 & 0.447 & 0.452 & 0.451 & 0.451 & 0.448 & 0.453 & 0.453 & 0.452 \\
\hline $\mathrm{H} 22$ & & 0.250 & 0.261 & 0.259 & 0.262 & 0.258 & 0.262 & 0.260 & 0.263 & 0.259 \\
\hline $\mathrm{H} 23$ & & 0.229 & 0.223 & 0.223 & 0.222 & 0.223 & 0.225 & 0.224 & 0.223 & 0.224 \\
\hline $\mathrm{H} 24$ & & 0.385 & 0.381 & 0.379 & 0.379 & 0.380 & 0.381 & 0.379 & 0.379 & 0.380 \\
\hline \multicolumn{11}{|c|}{ SS } \\
\hline N7 & & -0.582 & -0.500 & -0.407 & -0.922 & -0.447 & -0.547 & -0.450 & -0.952 & -0.484 \\
\hline $\mathrm{C} 9$ & & 0.664 & 0.500 & 0.232 & 0.674 & 0.194 & 0.453 & 0.182 & 0.629 & 0.128 \\
\hline $\mathrm{H} 12$ & & 0.047 & 0.060 & -0.003 & 0.018 & 0.010 & 0.013 & -0.052 & -0.021 & -0.043 \\
\hline $\mathrm{C} 2$ & & 0.762 & 0.500 & 0.376 & 0.696 & 0.451 & 0.500 & 0.373 & 0.683 & 0.449 \\
\hline H11 & & 0.303 & 0.300 & 0.253 & 0.475 & 0.218 & 0.300 & 0.256 & 0.470 & 0.220 \\
\hline $\mathrm{O} 10$ & & -0.515 & -0.500 & -0.310 & -0.445 & -0.183 & -0.500 & -0.309 & -0.450 & -0.173 \\
\hline N25 & & -0.535 & -0.500 & -0.282 & -0.636 & -0.383 & -0.500 & -0.280 & -0.639 & -0.377 \\
\hline Redistributed ch & $\left(q_{0}\right)$ & $\mathrm{n} / \mathrm{a}$ & 0.047 & 0.047 & 0.047 & 0.047 & 0.093 & 0.093 & 0.093 & 0.093 \\
\hline Link atom & & $\mathrm{n} / \mathrm{a}$ & 0.109 & 0.142 & 0.153 & 0.154 & 0.072 & 0.107 & 0.114 & 0.122 \\
\hline
\end{tabular}


D. Ace-His ${ }^{\delta}-\mathrm{NMe}$ (deprotonated at the delta position)

\begin{tabular}{|c|c|c|c|c|c|}
\hline Atom & QM & $\mathrm{RC}$ & $\begin{array}{l}\text { PBRC } \\
(\mathrm{SCT})\end{array}$ & RCD & $\begin{array}{r}\text { PBRCD } \\
(\mathrm{SCT})\end{array}$ \\
\hline \multicolumn{6}{|c|}{ PS } \\
\hline $\mathrm{C} 13$ & -0.397 & -0.407 & -0.406 & -0.371 & -0.372 \\
\hline $\mathrm{C} 14$ & 0.406 & 0.437 & 0.435 & 0.427 & 0.427 \\
\hline N15 & -0.550 & -0.573 & -0.574 & -0.571 & -0.572 \\
\hline $\mathrm{C} 16$ & -0.301 & -0.335 & -0.331 & -0.328 & -0.324 \\
\hline C17 & 0.198 & 0.220 & 0.224 & 0.220 & 0.224 \\
\hline N18 & -0.339 & -0.342 & -0.347 & -0.341 & -0.346 \\
\hline H19 & 0.113 & 0.124 & 0.127 & 0.123 & 0.126 \\
\hline $\mathrm{H} 20$ & 0.138 & 0.135 & 0.133 & 0.135 & 0.133 \\
\hline $\mathrm{H} 21$ & 0.222 & 0.234 & 0.229 & 0.234 & 0.230 \\
\hline $\mathrm{H} 22$ & 0.116 & 0.110 & 0.110 & 0.111 & 0.111 \\
\hline $\mathrm{H} 23$ & 0.345 & 0.346 & 0.347 & 0.347 & 0.348 \\
\hline \multicolumn{6}{|c|}{ SS } \\
\hline $\mathrm{N} 7$ & -0.573 & -0.500 & -0.361 & -0.547 & -0.403 \\
\hline C9 & 0.655 & 0.500 & 0.252 & 0.453 & 0.202 \\
\hline H12 & 0.101 & 0.060 & 0.098 & 0.013 & 0.049 \\
\hline $\mathrm{C} 2$ & 0.804 & 0.500 & 0.379 & 0.500 & 0.378 \\
\hline H11 & 0.333 & 0.300 & 0.211 & 0.300 & 0.212 \\
\hline $\mathrm{O} 10$ & -0.535 & -0.500 & -0.371 & -0.500 & -0.371 \\
\hline N24 & -0.631 & -0.500 & -0.348 & -0.500 & -0.347 \\
\hline $\begin{array}{l}\text { Redistributed } \\
\text { charge }\left(q_{0}\right)\end{array}$ & $\mathrm{n} / \mathrm{a}$ & 0.047 & 0.047 & 0.093 & 0.093 \\
\hline Link atom (HL) & $\mathrm{n} / \mathrm{a}$ & 0.050 & 0.053 & 0.013 & 0.016 \\
\hline
\end{tabular}


E. Ace-His ${ }^{\varepsilon}$-NMe (deprotonated at the epsilon position)

\begin{tabular}{|c|c|c|c|c|c|}
\hline Atom & QM & $\mathrm{RC}$ & $\begin{array}{l}\text { PBRC } \\
(\mathrm{SCT})\end{array}$ & $\mathrm{RCD}$ & $\begin{array}{r}\text { PBRCD } \\
(\mathrm{SCT})\end{array}$ \\
\hline \multicolumn{6}{|c|}{ PS } \\
\hline $\mathrm{C} 13$ & -0.402 & -0.504 & -0.503 & -0.469 & -0.469 \\
\hline $\mathrm{C} 14$ & -0.040 & 0.182 & 0.173 & 0.176 & 0.168 \\
\hline N15 & -0.280 & -0.447 & -0.442 & -0.447 & -0.442 \\
\hline $\mathrm{C} 16$ & 0.091 & 0.002 & 0.003 & 0.004 & 0.004 \\
\hline $\mathrm{C} 17$ & 0.241 & 0.257 & 0.256 & 0.260 & 0.259 \\
\hline N18 & -0.564 & -0.539 & -0.541 & -0.537 & -0.540 \\
\hline H19 & 0.134 & 0.178 & 0.167 & 0.178 & 0.168 \\
\hline $\mathrm{H} 20$ & 0.155 & 0.164 & 0.170 & 0.163 & 0.169 \\
\hline $\mathrm{H} 21$ & 0.300 & 0.398 & 0.401 & 0.400 & 0.403 \\
\hline $\mathrm{H} 22$ & 0.112 & 0.129 & 0.126 & 0.129 & 0.126 \\
\hline $\mathrm{H} 23$ & 0.099 & 0.098 & 0.098 & 0.099 & 0.099 \\
\hline \multicolumn{6}{|c|}{ SS } \\
\hline N7 & -0.874 & -0.500 & -0.373 & -0.547 & -0.415 \\
\hline C9 & 0.591 & 0.500 & 0.255 & 0.453 & 0.206 \\
\hline H12 & 0.038 & 0.060 & 0.078 & 0.013 & 0.029 \\
\hline $\mathrm{C} 2$ & 0.915 & 0.500 & 0.370 & 0.500 & 0.367 \\
\hline H11 & 0.361 & 0.300 & 0.212 & 0.300 & 0.214 \\
\hline $\mathrm{O} 10$ & -0.510 & -0.500 & -0.330 & -0.500 & -0.329 \\
\hline $\mathrm{N} 24$ & -0.542 & -0.500 & -0.352 & -0.500 & -0.350 \\
\hline $\begin{array}{l}\text { Redistributed } \\
\text { charge }\left(q_{0}\right)\end{array}$ & $\mathrm{n} / \mathrm{a}$ & 0.047 & 0.047 & 0.093 & 0.093 \\
\hline Link atom (HL) & $\mathrm{n} / \mathrm{a}$ & 0.082 & 0.091 & 0.044 & 0.054 \\
\hline
\end{tabular}


F. Ace-Tyr-NMe

\begin{tabular}{|c|c|c|c|c|c|}
\hline Atom & $\mathrm{QM}$ & $\mathrm{RC}$ & $\begin{array}{l}\text { PBRC } \\
(\mathrm{SCT})\end{array}$ & RCD & $\begin{array}{r}\text { PBRCD } \\
(\mathrm{SCT})\end{array}$ \\
\hline \multicolumn{6}{|c|}{ PS } \\
\hline $\mathrm{C} 13$ & -0.245 & -0.437 & -0.455 & -0.398 & -0.417 \\
\hline C14 & 0.014 & 0.124 & 0.140 & 0.112 & 0.130 \\
\hline $\mathrm{C} 15$ & -0.157 & -0.196 & -0.206 & -0.191 & -0.201 \\
\hline C16 & -0.038 & -0.126 & -0.130 & -0.124 & -0.128 \\
\hline $\mathrm{C} 17$ & -0.360 & -0.340 & -0.340 & -0.342 & -0.342 \\
\hline $\mathrm{C} 18$ & -0.488 & -0.449 & -0.454 & -0.448 & -0.453 \\
\hline C19 & 0.522 & 0.492 & 0.493 & 0.496 & 0.496 \\
\hline $\mathrm{O} 20$ & -0.587 & -0.588 & -0.590 & -0.588 & -0.589 \\
\hline $\mathrm{H} 21$ & 0.094 & 0.144 & 0.147 & 0.143 & 0.145 \\
\hline $\mathrm{H} 22$ & 0.129 & 0.187 & 0.189 & 0.186 & 0.187 \\
\hline $\mathrm{H} 23$ & 0.180 & 0.175 & 0.177 & 0.176 & 0.179 \\
\hline $\mathrm{H} 24$ & 0.147 & 0.161 & 0.160 & 0.162 & 0.162 \\
\hline $\mathrm{H} 25$ & 0.201 & 0.202 & 0.202 & 0.203 & 0.203 \\
\hline $\mathrm{H} 26$ & 0.191 & 0.190 & 0.190 & 0.190 & 0.191 \\
\hline $\mathrm{H} 27$ & 0.408 & 0.410 & 0.410 & 0.410 & 0.410 \\
\hline \multicolumn{6}{|c|}{ SS } \\
\hline N7 & -0.681 & -0.500 & -0.369 & -0.547 & -0.412 \\
\hline C9 & 0.735 & 0.500 & 0.247 & 0.453 & 0.196 \\
\hline H12 & 0.073 & 0.060 & 0.065 & 0.013 & 0.017 \\
\hline $\mathrm{C} 2$ & 0.841 & 0.500 & 0.398 & 0.500 & 0.395 \\
\hline H11 & 0.342 & 0.300 & 0.231 & 0.300 & 0.233 \\
\hline O10 & -0.540 & -0.500 & -0.362 & -0.500 & -0.360 \\
\hline $\mathrm{N} 28$ & -0.666 & -0.500 & -0.349 & -0.500 & -0.349 \\
\hline $\begin{array}{l}\text { Redistributed } \\
\text { charge }\left(q_{0}\right)\end{array}$ & $\mathrm{n} / \mathrm{a}$ & 0.047 & 0.047 & 0.093 & 0.093 \\
\hline Link atom (HL) & $\mathrm{n} / \mathrm{a}$ & 0.052 & 0.066 & 0.014 & 0.028 \\
\hline
\end{tabular}


G. Deprotonated Ace-Tyr-NMe

\begin{tabular}{|c|c|c|c|c|c|}
\hline Atom & $\mathrm{QM}$ & $\mathrm{RC}$ & $\begin{array}{l}\text { PBRC } \\
(\mathrm{SCT})\end{array}$ & $\mathrm{RCD}$ & $\begin{array}{r}\text { PBRCD } \\
(\mathrm{SCT})\end{array}$ \\
\hline \multicolumn{6}{|c|}{ PS } \\
\hline $\mathrm{C} 13$ & -0.208 & -0.348 & -0.394 & -0.302 & -0.347 \\
\hline C14 & -0.200 & -0.111 & -0.104 & -0.127 & -0.119 \\
\hline $\mathrm{C} 15$ & -0.088 & -0.090 & -0.089 & -0.084 & -0.084 \\
\hline C16 & -0.012 & -0.063 & -0.050 & -0.061 & -0.049 \\
\hline $\mathrm{C} 17$ & -0.545 & -0.574 & -0.587 & -0.574 & -0.586 \\
\hline $\mathrm{C} 18$ & -0.569 & -0.594 & -0.616 & -0.592 & -0.614 \\
\hline C19 & 0.753 & 0.754 & 0.766 & 0.757 & 0.769 \\
\hline $\mathrm{O} 20$ & -0.730 & -0.740 & -0.740 & -0.738 & -0.738 \\
\hline $\mathrm{H} 21$ & 0.076 & 0.107 & 0.128 & 0.105 & 0.125 \\
\hline $\mathrm{H} 22$ & 0.104 & 0.137 & 0.157 & 0.135 & 0.154 \\
\hline $\mathrm{H} 23$ & 0.144 & 0.123 & 0.123 & 0.124 & 0.125 \\
\hline $\mathrm{H} 24$ & 0.107 & 0.109 & 0.106 & 0.110 & 0.107 \\
\hline $\mathrm{H} 25$ & 0.136 & 0.148 & 0.153 & 0.148 & 0.153 \\
\hline $\mathrm{H} 26$ & 0.134 & 0.149 & 0.156 & 0.149 & 0.155 \\
\hline \multicolumn{6}{|c|}{ SS } \\
\hline N7 & -0.655 & -0.500 & -0.343 & -0.547 & -0.385 \\
\hline C9 & 0.790 & 0.500 & 0.282 & 0.453 & 0.232 \\
\hline H12 & 0.059 & 0.060 & 0.114 & 0.013 & 0.066 \\
\hline $\mathrm{C} 2$ & 0.800 & 0.500 & 0.323 & 0.500 & 0.320 \\
\hline H11 & 0.321 & 0.300 & 0.193 & 0.300 & 0.195 \\
\hline $\mathrm{O} 10$ & -0.577 & -0.500 & -0.404 & -0.500 & -0.402 \\
\hline $\mathrm{N} 27$ & -0.686 & -0.500 & -0.305 & -0.500 & -0.307 \\
\hline $\begin{array}{l}\text { Redistributed } \\
\text { charge }\left(q_{0}\right)\end{array}$ & $\mathrm{n} / \mathrm{a}$ & 0.047 & 0.047 & 0.093 & 0.093 \\
\hline Link atom (HL) & $\mathrm{n} / \mathrm{a}$ & -0.009 & -0.008 & -0.051 & -0.050 \\
\hline
\end{tabular}


H. Ace-Glu-NMe

\begin{tabular}{|c|c|c|c|c|c|}
\hline Atom & QM & $\mathrm{RC}$ & $\begin{array}{l}\text { PBRC } \\
(\mathrm{SCT})\end{array}$ & $\mathrm{RCD}$ & $\begin{array}{r}\text { PBRCD } \\
(\mathrm{SCT})\end{array}$ \\
\hline \multicolumn{6}{|c|}{ PS } \\
\hline $\mathrm{C} 13$ & 0.148 & -0.027 & -0.025 & -0.003 & -0.001 \\
\hline $\mathrm{C} 14$ & -0.439 & -0.250 & -0.233 & -0.252 & -0.234 \\
\hline $\mathrm{C} 15$ & 0.788 & 0.734 & 0.727 & 0.731 & 0.724 \\
\hline O16 & -0.493 & -0.491 & -0.493 & -0.488 & -0.490 \\
\hline $\mathrm{C} 17$ & -0.630 & -0.606 & -0.605 & -0.604 & -0.603 \\
\hline $\mathrm{H} 18$ & 0.045 & 0.057 & 0.051 & 0.060 & 0.053 \\
\hline H19 & 0.002 & 0.047 & 0.040 & 0.050 & 0.043 \\
\hline $\mathrm{H} 20$ & 0.105 & 0.059 & 0.053 & 0.063 & 0.057 \\
\hline $\mathrm{H} 21$ & 0.078 & 0.070 & 0.061 & 0.073 & 0.064 \\
\hline $\mathrm{H} 28$ & 0.438 & 0.427 & 0.428 & 0.426 & 0.427 \\
\hline \multicolumn{6}{|c|}{ SS } \\
\hline N7 & -0.714 & -0.500 & -0.371 & -0.547 & -0.414 \\
\hline C9 & 0.676 & 0.500 & 0.238 & 0.453 & 0.187 \\
\hline H12 & 0.127 & 0.060 & 0.046 & 0.013 & -0.003 \\
\hline $\mathrm{C} 2$ & 0.847 & 0.500 & 0.409 & 0.500 & 0.407 \\
\hline H11 & 0.365 & 0.300 & 0.242 & 0.300 & 0.245 \\
\hline $\mathrm{O} 10$ & -0.525 & -0.500 & -0.344 & -0.500 & -0.342 \\
\hline $\mathrm{N} 22$ & -0.408 & -0.500 & -0.360 & -0.500 & -0.360 \\
\hline $\begin{array}{l}\text { Redistributed } \\
\text { charge }\left(q_{0}\right)\end{array}$ & $\mathrm{n} / \mathrm{a}$ & 0.047 & 0.047 & 0.093 & 0.093 \\
\hline Link atom (HL) & $\mathrm{n} / \mathrm{a}$ & -0.020 & -0.006 & -0.055 & -0.041 \\
\hline
\end{tabular}


I. Deprotonated Ace-Glu-NMe

\begin{tabular}{|c|c|c|c|c|c|}
\hline Atom & QM & $\mathrm{RC}$ & $\begin{array}{l}\text { PBRC } \\
(\mathrm{SCT})\end{array}$ & $\mathrm{RCD}$ & $\begin{array}{r}\text { PBRCD } \\
(\mathrm{SCT})\end{array}$ \\
\hline \multicolumn{6}{|c|}{ PS } \\
\hline $\mathrm{C} 13$ & 0.039 & -0.294 & -0.287 & -0.256 & -0.249 \\
\hline $\mathrm{C} 14$ & -0.208 & -0.016 & -0.011 & -0.025 & -0.019 \\
\hline $\mathrm{C} 15$ & 0.726 & 0.780 & 0.777 & 0.777 & 0.774 \\
\hline O16 & -0.742 & -0.773 & -0.771 & -0.769 & -0.767 \\
\hline $\mathrm{C} 17$ & -0.730 & -0.756 & -0.752 & -0.754 & -0.750 \\
\hline $\mathrm{H} 18$ & -0.004 & 0.068 & 0.077 & 0.068 & 0.077 \\
\hline H19 & 0.042 & 0.110 & 0.104 & 0.110 & 0.104 \\
\hline $\mathrm{H} 20$ & 0.013 & -0.034 & -0.038 & -0.028 & -0.033 \\
\hline $\mathrm{H} 21$ & 0.023 & -0.050 & -0.052 & -0.047 & -0.049 \\
\hline \multicolumn{6}{|c|}{ SS } \\
\hline N7 & -0.785 & -0.500 & -0.315 & -0.547 & -0.356 \\
\hline C9 & 0.668 & 0.500 & 0.296 & 0.453 & 0.246 \\
\hline H12 & 0.049 & 0.060 & 0.117 & 0.013 & 0.068 \\
\hline $\mathrm{C} 2$ & 0.909 & 0.500 & 0.323 & 0.500 & 0.322 \\
\hline H11 & 0.331 & 0.300 & 0.208 & 0.300 & 0.208 \\
\hline $\mathrm{O} 10$ & -0.551 & -0.500 & -0.392 & -0.500 & -0.390 \\
\hline $\mathrm{N} 22$ & -0.634 & -0.500 & -0.377 & -0.500 & -0.377 \\
\hline $\begin{array}{c}\text { Redistributed } \\
\text { charge }\left(q_{0}\right)\end{array}$ & $\mathrm{n} / \mathrm{a}$ & 0.047 & 0.047 & 0.093 & 0.093 \\
\hline Link atom (HL) & $\mathrm{n} / \mathrm{a}$ & -0.036 & -0.046 & -0.076 & -0.086 \\
\hline
\end{tabular}

a. The side chain is the PS, and the backbone is the SS. Only the SCT results are shown for the PBRC and PBRCD calculations. The QM level is HF/MIDI!, and the MM force field is OPLS-AA. The iterative procedure for polarization was initiated by using OPLS-AA charges for the embedded-QM calculations. For the PS atoms, the QM ESP charges are listed. 
Table S8. Atom types, full-QM optimized coordinates (at the B3LYP/6-31+G* level), and connectivities for the amino acids in the tinker .xyz file format

A. Ace-Lys-NMe

\begin{tabular}{|c|c|c|c|c|c|c|c|c|c|}
\hline \multicolumn{10}{|c|}{34 Lysine } \\
\hline 10 & & -0.863483 & -0.799142 & 0.335592 & 1 & 2 & 4 & 5 & 6 \\
\hline & C & -0.812226 & -0.780191 & 1.845748 & 82 & 1 & 3 & 7 & \\
\hline 3 & 0 & -0.262482 & -1.671463 & 2.498985 & 83 & 2 & & & \\
\hline 4 & $\mathrm{HC}$ & $\odot .160268$ & -0.733078 & $-\odot .048233$ & 6 & 1 & & & \\
\hline 5 & $\mathrm{HC}$ & -1.455629 & 0.014335 & $-\odot .093990$ & 6 & 1 & & & \\
\hline 6 & $\mathrm{HC}$ & -1.273713 & -1.758013 & ๑.0๑5328 & 6 & 1 & & & \\
\hline 7 & $\mathrm{~N}$ & -1.405582 & 0.283732 & 2.473975 & 85 & 2 & 8 & 11 & \\
\hline 8 & CT & -1.275181 & 0.507098 & 3.915685 & 74 & 7 & 9 & 12 & 13 \\
\hline 9 & C & -2.302011 & $-\odot .350554$ & 4.701430 & 82 & 8 & 10 & 29 & \\
\hline 10 & 0 & -3.266082 & $\odot .157969$ & 5.328262 & 83 & 9 & & & \\
\hline 11 & $\mathrm{H}$ & -1.868813 & 0.990113 & 1.916588 & 88 & 7 & & & \\
\hline 12 & $\mathrm{HC}$ & -0.273852 & 0.156785 & 4.195360 & 6 & 8 & & & \\
\hline 13 & CT & -1.409838 & 1.997244 & 4.244763 & 2 & 8 & 14 & 18 & 19 \\
\hline 14 & CT & -0.962716 & 2.346902 & 5.685415 & 2 & 13 & 15 & 20 & 21 \\
\hline 15 & CT & -1.712415 & 3.539291 & 6.319607 & 2 & 14 & 16 & 22 & 23 \\
\hline 16 & CT & -2.954030 & 3.166761 & 7.131544 & 155 & 15 & 17 & 24 & 25 \\
\hline 17 & N3 & -4.022803 & 2.491731 & 6.298744 & 146 & 16 & 26 & 27 & 28 \\
\hline 18 & $\mathrm{HC}$ & -2.447915 & 2.305309 & 4.061199 & 6 & 13 & & & \\
\hline 19 & $\mathrm{HC}$ & -0.797218 & 2.562527 & 3.533170 & 6 & 13 & & & \\
\hline 20 & $\mathrm{HC}$ & $\odot .108214$ & 2.574817 & 5.666713 & 6 & 14 & & & \\
\hline 21 & $\mathrm{HC}$ & -1.065346 & 1.473581 & 6.342597 & 6 & 14 & & & \\
\hline 22 & $\mathrm{HC}$ & -1.968804 & 4.287376 & 5.556048 & 6 & 15 & & & \\
\hline 23 & $\mathrm{HC}$ & -1.051765 & 4.055028 & 7.026560 & 6 & 15 & & & \\
\hline 24 & $\mathrm{HC}$ & -3.410445 & 4.049170 & 7.588191 & 6 & 16 & & & \\
\hline 25 & $\mathrm{HC}$ & -2.704040 & 2.455963 & 7.924128 & 6 & 16 & & & \\
\hline 26 & H3 & -4.830295 & 2.235313 & 6.875869 & 151 & 17 & & & \\
\hline 27 & H3 & -3.663442 & 1.584928 & 5.839606 & 151 & 17 & & & \\
\hline 28 & H3 & -4.367423 & 3.113536 & 5.561143 & 151 & 17 & & & \\
\hline 29 & $\mathrm{~N}$ & -2.100381 & -1.664017 & 4.668699 & 85 & 9 & 30 & 31 & \\
\hline 30 & CT & -2.995326 & -2.626316 & 5.308493 & 89 & 29 & 32 & 33 & 34 \\
\hline 31 & $\mathrm{H}$ & -1.331369 & -1.994787 & 4.079158 & 88 & 29 & & & \\
\hline 32 & $\mathrm{HC}$ & -2.573899 & -3.623722 & 5.177141 & 6 & 30 & & & \\
\hline 33 & $\mathrm{HC}$ & -3.992081 & -2.590685 & 4.857369 & 6 & 30 & & & \\
\hline 34 & $\mathrm{HC}$ & -3.086064 & -2.408949 & 6.376267 & 6 & 30 & & & \\
\hline
\end{tabular}


B. Ace-His ${ }^{+}-\mathrm{NMe}$

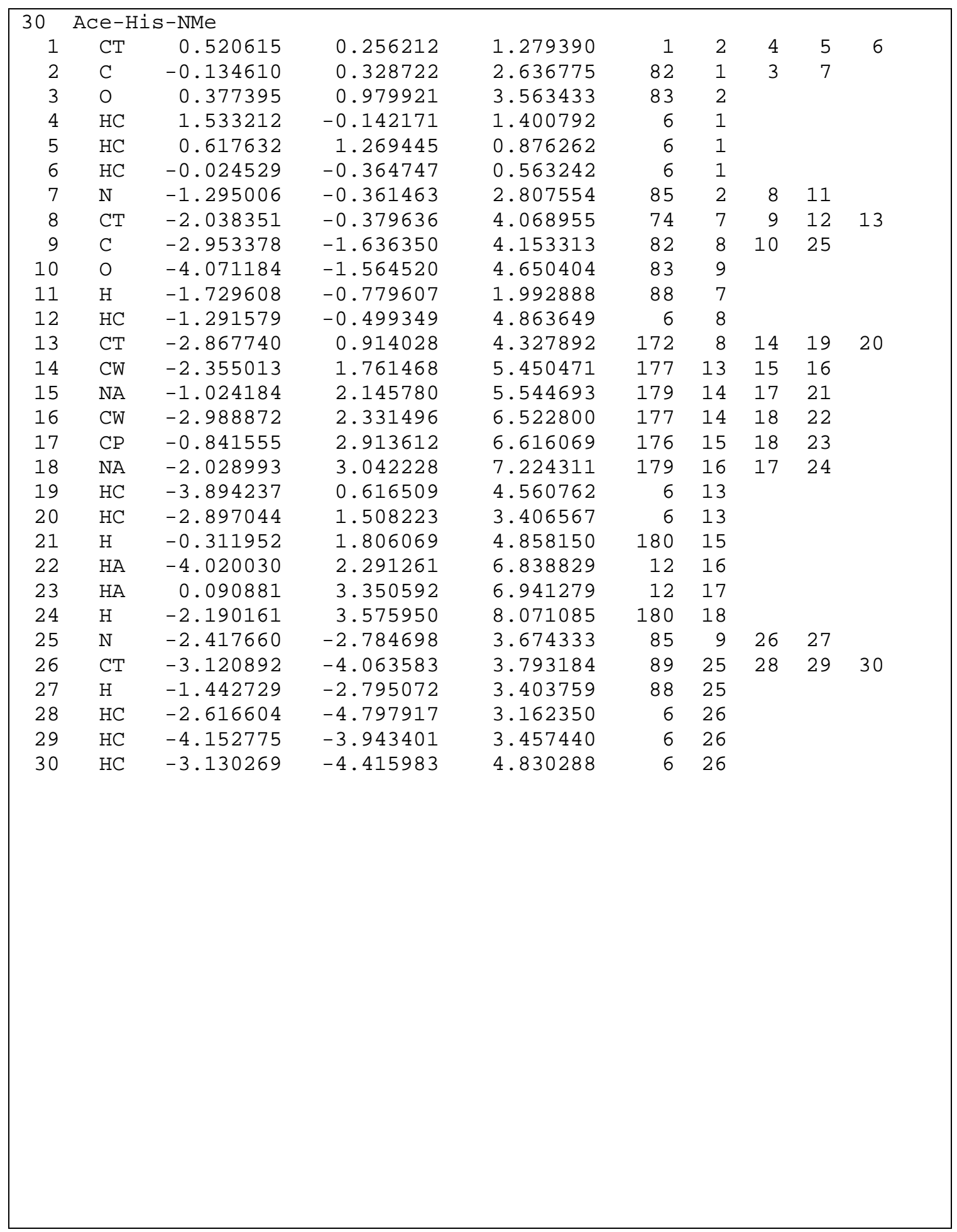


C. Ace-Glu-NMe

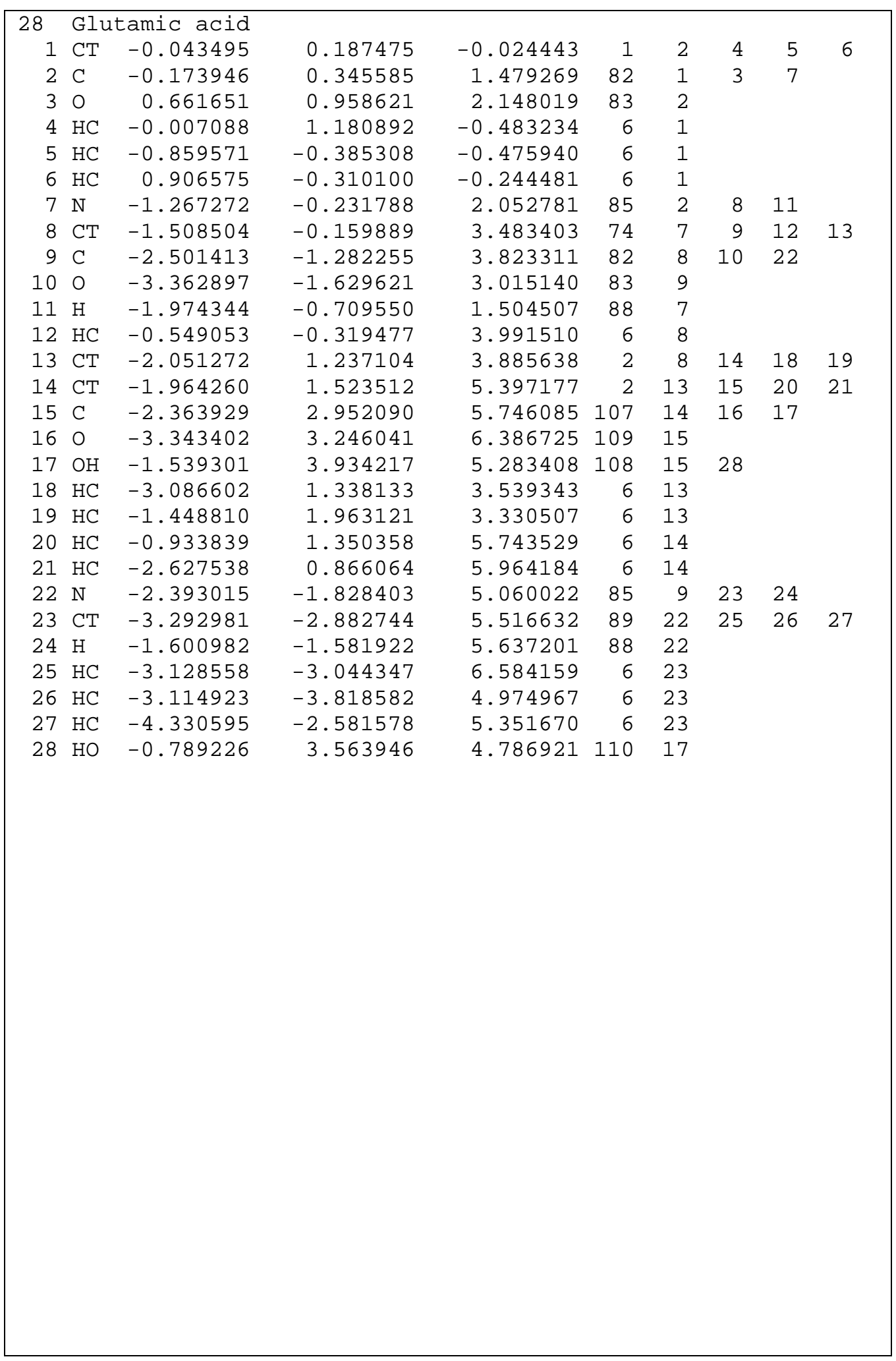


D. Ace-Tyr-NMe

\begin{tabular}{|c|c|c|c|c|c|c|c|c|c|}
\hline \multicolumn{10}{|c|}{33 Ace-tyr-NMe } \\
\hline 1 & CT & $\odot .149943$ & $\odot .309550$ & ๑. 029950 & 1 & 2 & 4 & 5 & 6 \\
\hline 2 & C & $-\odot .045784$ & 0.424409 & 1.532138 & 82 & 1 & 3 & 7 & \\
\hline 3 & 0 & 0.751009 & 1.035053 & 2.247491 & 83 & 2 & & & \\
\hline 4 & $\mathrm{HC}$ & 1.115644 & -0.168843 & -0.163438 & 6 & 1 & & & \\
\hline 5 & $\mathrm{HC}$ & $\odot .190976$ & 1.315961 & -0.399246 & 6 & 1 & & & \\
\hline 6 & $\mathrm{HC}$ & -0.638394 & -0.260740 & $-\odot .471717$ & 6 & 1 & & & \\
\hline 7 & $\mathrm{~N}$ & -1.154027 & -0.184635 & 2.038666 & 85 & 2 & 8 & 11 & \\
\hline 8 & CT & -1.446145 & -0.202179 & 3.459674 & 74 & 7 & 9 & 12 & 13 \\
\hline 9 & C & -2.347279 & -1.420296 & 3.716607 & 82 & 8 & 10 & 28 & \\
\hline 10 & 0 & -3.039567 & -1.903236 & 2.816796 & 83 & 9 & & & \\
\hline 11 & $\mathrm{H}$ & -1.779902 & -0.730068 & 1.455360 & 88 & 7 & & & \\
\hline 12 & $\mathrm{HC}$ & $-\odot .498409$ & $-\odot .308157$ & 3.999138 & 6 & 8 & & & \\
\hline 13 & CT & -2.120427 & 1.130985 & 3.912177 & 15 & 8 & 14 & 21 & 22 \\
\hline 14 & CA & -2.226836 & 1.305878 & 5.411495 & 11 & 13 & 15 & 16 & \\
\hline 15 & CA & -1.077788 & 1.543293 & 6.187725 & 11 & 14 & 17 & 23 & \\
\hline 16 & CA & -3.458893 & 1.241110 & 6.074607 & 11 & 14 & 18 & 24 & \\
\hline 17 & CA & -1.151173 & 1.700301 & 7.570769 & 11 & 15 & 19 & 25 & \\
\hline 18 & CA & -3.550883 & 1.402491 & 7.460371 & 11 & 16 & 19 & 26 & \\
\hline 19 & CA & -2.393759 & 1.630178 & 8.211190 & 28 & 17 & 18 & 20 & \\
\hline 20 & $\mathrm{OH}$ & -2.411161 & 1.796215 & 9.573062 & 29 & 19 & 27 & & \\
\hline 21 & $\mathrm{HC}$ & -3.110692 & 1.194539 & 3.445721 & 6 & 13 & & & \\
\hline 22 & $\mathrm{HC}$ & -1.506133 & 1.932699 & 3.488499 & 6 & 13 & & & \\
\hline 23 & HA & $-\odot .109422$ & 1.621333 & 5.697213 & 12 & 15 & & & \\
\hline 24 & HA & -4.367699 & 1.065867 & 5.502711 & 12 & 16 & & & \\
\hline 25 & HA & $-\odot .261084$ & 1.890870 & 8.163065 & 12 & 17 & & & \\
\hline 26 & $\mathrm{HA}$ & -4.521967 & 1.354929 & 7.951022 & 12 & 18 & & & \\
\hline 27 & $\mathrm{HO}$ & -3.323595 & 1.755679 & 9.899566 & 30 & 20 & & & \\
\hline 28 & $\mathrm{~N}$ & -2.329480 & -1.916323 & 4.977570 & 85 & 9 & 29 & 30 & \\
\hline 29 & CT & -3.216342 & -2.994198 & 5.395496 & 89 & 28 & 31 & 32 & 33 \\
\hline 30 & $\mathrm{H}$ & -1.829350 & -1.398359 & 5.688388 & 88 & 28 & & & \\
\hline 31 & $\mathrm{HC}$ & -2.855979 & -3.396934 & 6.345013 & 6 & 29 & & & \\
\hline 32 & $\mathrm{HC}$ & -3.208341 & -3.783788 & 4.640695 & 6 & 29 & & & \\
\hline 33 & $\mathrm{HC}$ & -4.249305 & -2.645425 & 5.519075 & 6 & 29 & & & \\
\hline
\end{tabular}


Table S9. Full-QM and QM/MM proton affinities for the small organic molecules (with $\left.\mathrm{QM}=\mathrm{B} 3 \mathrm{LYP} / 6-31+\mathrm{G}^{*}\right)^{a}$

\begin{tabular}{|c|c|c|c|c|c|c|c|c|c|c|}
\hline \multirow{2}{*}{$\begin{array}{l}\text { Molecule } \\
\text { (SS-PS) }\end{array}$} & \multirow[t]{2}{*}{ QM } & \multirow{2}{*}{$\begin{array}{l}\text { Initial } \\
\text { charge }\end{array}$} & \multirow[t]{2}{*}{$\mathrm{RC}$} & \multicolumn{3}{|c|}{ PBRC } & \multirow[t]{2}{*}{$\mathrm{RCD}$} & \multicolumn{3}{|c|}{ PBRCD } \\
\hline & & & & $\mathrm{SCT}^{b}$ & $\mathrm{BT}^{c}$ & EEM & & $\mathrm{SCT}^{b}$ & $\mathrm{BT}^{c}$ & EEM \\
\hline \multirow[t]{2}{*}{$\mathrm{CH}_{3}-\mathrm{CH}_{2} \mathrm{OH}$} & 381.5 & OPLS-AA & 389.7 & 389.5 & 389.3 & 389.6 & 393.0 & 392.7 & 392.6 & 392.9 \\
\hline & & ESP & 387.2 & 387.0 & 386.9 & 387.1 & 388.3 & 388.1 & 387.9 & 388.2 \\
\hline \multirow{2}{*}{$\mathrm{CH}_{3}-\mathrm{CH}_{2} \mathrm{SH}$} & 356.5 & OPLS-AA & 360.8 & 360.1 & 359.8 & 360.5 & 362.6 & 361.9 & 361.5 & 362.3 \\
\hline & & ESP & 359.4 & 358.8 & 358.4 & 359.1 & 360.0 & 359.4 & 359.0 & 359.7 \\
\hline \multirow[t]{2}{*}{$\mathrm{CH}_{3}-\mathrm{CH}_{2} \mathrm{NH}_{3}^{+}$} & 225.8 & OPLS-AA & 225.7 & 226.2 & 226.5 & 225.9 & 228.6 & 229.0 & 229.3 & 228.8 \\
\hline & & ESP & 223.6 & 224.1 & 224.4 & 223.8 & 224.5 & 225.0 & 225.3 & 224.7 \\
\hline \multirow[t]{2}{*}{$\mathrm{CH}_{3}-\mathrm{CH}_{2} \mathrm{COOH}$} & 349.2 & OPLS-AA & 351.5 & 350.9 & 350.3 & 351.2 & 353.3 & 352.6 & 351.8 & 353.0 \\
\hline & & ESP & 350.2 & 349.6 & 349.2 & 349.9 & 350.7 & 350.1 & 349.8 & 350.5 \\
\hline \multirow[t]{2}{*}{$\mathrm{CF}_{3}-\mathrm{CH}_{2} \mathrm{OH}$} & 360.4 & OPLS-AA & 383.2 & 383.0 & $\mathrm{n} / \mathrm{a}$ & $\mathrm{n} / \mathrm{a}$ & 368.1 & 368.0 & $\mathrm{n} / \mathrm{a}$ & $\mathrm{n} / \mathrm{a}$ \\
\hline & & ESP & 376.0 & 375.8 & $\mathrm{n} / \mathrm{a}$ & $\mathrm{n} / \mathrm{a}$ & 364.3 & 364.1 & $\mathrm{n} / \mathrm{a}$ & $\mathrm{n} / \mathrm{a}$ \\
\hline \multirow[t]{2}{*}{$\mathrm{CH}_{3} \mathrm{OH}-\mathrm{CH}_{2} \mathrm{OH}$} & 378.4 & OPLS-AA & 388.2 & 381.7 & 382.8 & 383.3 & 384.7 & 378.1 & 378.7 & 379.9 \\
\hline & & ESP & 386.4 & 379.9 & 380.7 & 381.6 & 380.8 & 374.0 & 374.0 & 376.2 \\
\hline \multirow[t]{2}{*}{$\mathrm{CH}_{2} \mathrm{OH}-\mathrm{CH}_{2} \mathrm{SH}$} & 351.9 & OPLS-AA & 358.5 & 354.5 & 355.3 & 356.2 & 356.6 & 352.6 & 353.0 & 354.4 \\
\hline & & ESP & 357.9 & 353.5 & 354.2 & 355.3 & 354.8 & 350.4 & 350.4 & 352.4 \\
\hline
\end{tabular}




\begin{tabular}{llllllllll} 
MUE (seven) ${ }^{d}$ & OPLS-AA & $5.2 / 7.7$ & $3.2 / 6.0$ & 3.4 & 3.9 & $5.9 / 6.2$ & $4.0 / 4.5$ & 3.9 & 4.6 \\
& ESP & $4.3 / 5.9$ & $2.2 / 4.1$ & 2.2 & 2.9 & $3.1 / 3.2$ & $2.9 / 3.0$ & 2.7 & 2.5 \\
MSE (seven) & & & & & & & & & \\
& OPLS-AA & $5.2 / 7.7$ & $3.2 / 6.0$ & 3.4 & 3.9 & $5.9 / 6.2$ & $3.9 / 4.4$ & 3.9 & 4.6 \\
& ESP & $3.5 / 5.3$ & $1.6 / 3.6$ & 1.7 & 2.2 & $2.6 / 2.8$ & $0.6 / 1.1$ & 0.5 \\
\hline
\end{tabular}

a. The QM level is B3LYP/6-31+G*, and the MM force field is OPLS-AA. The iterative procedure for polarization was initiated by using the OPLS-AA or ESP charges for the embedded-QM calculations.
b. QEq model with SCT of Rappé and Goddard
c. QEq model of Bakowies and Thiel
d. Mean unsigned error excluding/including $\mathrm{CF}_{3}-\mathrm{CH}_{2} \mathrm{OH}$
e. Mean signed error excluding/including $\mathrm{CF}_{3}-\mathrm{CH}_{2} \mathrm{O}$ 
Table S10. QM/MM optimized Q1-M1 bond distances $(\AA)$ in comparison with full-QM results for small organic molecules (with QM $\left.=\mathrm{B} 3 \mathrm{LYP} / 6-31+\mathrm{G}^{*}\right)^{a}$

\begin{tabular}{|c|c|c|c|c|c|c|c|c|c|c|}
\hline \multirow{2}{*}{$\begin{array}{l}\text { Molecule } \\
\text { (SS-PS) }\end{array}$} & \multirow[t]{2}{*}{$\mathrm{QM}$} & \multirow{2}{*}{$\begin{array}{l}\text { Initial } \\
\text { charge }\end{array}$} & \multirow[t]{2}{*}{$\mathrm{RC}$} & \multicolumn{3}{|c|}{ PBRC } & \multirow[t]{2}{*}{ RCD } & \multicolumn{3}{|c|}{ PBRCD } \\
\hline & & & & $\mathrm{SCT}^{b}$ & $\mathrm{BT}^{C}$ & EEM & & $\mathrm{SCT}^{b}$ & $\mathrm{BT}^{C}$ & EEM \\
\hline \multirow[t]{2}{*}{$\mathrm{CH}_{3}-\mathrm{CH}_{2} \mathrm{OH}$} & 1.519 & OPLS-AA & 1.526 & 1.526 & 1.526 & 1.526 & 1.518 & 1.518 & 1.518 & 1.518 \\
\hline & & ESP & 1.531 & 1.531 & 1.531 & 1.531 & 1.529 & 1.529 & 1.529 & 1.529 \\
\hline \multirow{2}{*}{$\mathrm{CH}_{3}-\mathrm{CH}_{2} \mathrm{SH}$} & 1.528 & OPLS-AA & 1.526 & 1.526 & 1.526 & 1.526 & 1.519 & 1.519 & 1.519 & 1.519 \\
\hline & & ESP & 1.531 & 1.531 & 1.531 & 1.531 & 1.529 & 1.529 & 1.529 & 1.529 \\
\hline \multirow[t]{2}{*}{$\mathrm{CH}_{3}-\mathrm{CH}_{2} \mathrm{NH}_{3}^{+}$} & 1.525 & OPLS-AA & 1.525 & 1.524 & 1.524 & 1.524 & 1.519 & 1.518 & 1.518 & 1.518 \\
\hline & & ESP & 1.529 & 1.528 & 1.528 & 1.529 & 1.527 & 1.527 & 1.526 & 1.527 \\
\hline \multirow[t]{2}{*}{$\mathrm{CH}_{3}-\mathrm{CH}_{2} \mathrm{COOH}$} & 1.528 & OPLS-AA & 1.528 & 1.528 & 1.528 & 1.528 & 1.522 & 1.522 & 1.522 & 1.522 \\
\hline & & ESP & 1.533 & 1.533 & 1.532 & 1.533 & 1.531 & 1.531 & 1.531 & 1.531 \\
\hline \multirow[t]{2}{*}{$\mathrm{CF}_{3}-\mathrm{CH}_{2} \mathrm{OH}$} & 1.521 & OPLS-AA & 1.557 & 1.557 & $\mathrm{n} / \mathrm{a}$ & $\mathrm{n} / \mathrm{a}$ & 1.582 & 1.582 & $\mathrm{n} / \mathrm{a}$ & $\mathrm{n} / \mathrm{a}$ \\
\hline & & ESP & 1.556 & 1.556 & $\mathrm{n} / \mathrm{a}$ & $\mathrm{n} / \mathrm{a}$ & 1.576 & 1.576 & $\mathrm{n} / \mathrm{a}$ & $\mathrm{n} / \mathrm{a}$ \\
\hline \multirow[t]{2}{*}{$\mathrm{CH}_{2} \mathrm{OH}-\mathrm{CH}_{2} \mathrm{OH}$} & 1.521 & OPLS-AA & 1.534 & 1.536 & 1.534 & 1.537 & 1.538 & 1.541 & 1.539 & 1.542 \\
\hline & & ESP & 1.537 & 1.538 & 1.536 & 1.540 & 1.544 & 1.546 & 1.544 & 1.547 \\
\hline \multirow[t]{2}{*}{$\mathrm{CH}_{2} \mathrm{OH}-\mathrm{CH}_{2} \mathrm{SH}$} & 1.523 & OPLS-AA & 1.535 & 1.537 & 1.535 & 1.538 & 1.539 & 1.541 & 1.540 & 1.542 \\
\hline & & ESP & 1.538 & 1.539 & 1.538 & 1.540 & 1.545 & 1.546 & 1.544 & 1.546 \\
\hline \multirow[t]{2}{*}{$\mathrm{CH}_{3}-\mathrm{CH}_{2} \mathrm{O}^{-}$} & 1.565 & OPLS-AA & 1.557 & 1.556 & 1.556 & 1.557 & 1.544 & 1.543 & 1.543 & 1.544 \\
\hline & & ESP & 1.566 & 1.566 & 1.565 & 1.566 & 1.562 & 1.561 & 1.561 & 1.562 \\
\hline
\end{tabular}




\begin{tabular}{|c|c|c|c|c|c|c|c|c|c|c|}
\hline \multirow[t]{2}{*}{$\mathrm{CH}_{3}-\mathrm{CH}_{2} \mathrm{~S}^{-}$} & 1.537 & OPLS-AA & 1.529 & 1.528 & 1.528 & 1.529 & 1.520 & 1.519 & 1.519 & 1.519 \\
\hline & & ESP & 1.536 & 1.535 & 1.534 & 1.536 & 1.533 & 1.532 & 1.532 & 1.532 \\
\hline \multirow[t]{2}{*}{$\mathrm{CH}_{3}-\mathrm{CH}_{2} \mathrm{NH}_{2}$} & 1.535 & OPLS-AA & 1.533 & 1.533 & 1.533 & 1.533 & 1.525 & 1.525 & 1.525 & 1.525 \\
\hline & & ESP & 1.539 & 1.539 & 1.539 & 1.539 & 1.537 & 1.537 & 1.536 & 1.537 \\
\hline \multirow[t]{2}{*}{$\mathrm{CH}_{3}-\mathrm{CH}_{2} \mathrm{COO}^{-}$} & 1.532 & OPLS-AA & 1.534 & 1.533 & 1.534 & 1.534 & 1.526 & 1.526 & 1.526 & 1.526 \\
\hline & & ESP & 1.539 & 1.539 & 1.539 & 1.539 & 1.537 & 1.536 & 1.536 & 1.537 \\
\hline \multirow[t]{2}{*}{$\mathrm{CF}_{3}-\mathrm{CH}_{2} \mathrm{O}^{-}$} & 1.551 & OPLS-AA & 1.610 & 1.610 & $\mathrm{n} / \mathrm{a}$ & $\mathrm{n} / \mathrm{a}$ & 1.662 & 1.661 & $\mathrm{n} / \mathrm{a}$ & $\mathrm{n} / \mathrm{a}$ \\
\hline & & ESP & 1.606 & 1.606 & $\mathrm{n} / \mathrm{a}$ & $\mathrm{n} / \mathrm{a}$ & 1.647 & 1.647 & $\mathrm{n} / \mathrm{a}$ & $\mathrm{n} / \mathrm{a}$ \\
\hline \multirow[t]{2}{*}{$\mathrm{CH}_{2} \mathrm{OH}-\mathrm{CH}_{2} \mathrm{O}^{-}$} & 1.548 & OPLS-AA & 1.574 & 1.579 & 1.577 & 1.581 & 1.585 & 1.591 & 1.589 & 1.592 \\
\hline & & ESP & 1.581 & 1.585 & 1.583 & 1.587 & 1.598 & 1.605 & 1.603 & 1.605 \\
\hline \multirow[t]{2}{*}{$\mathrm{CH}_{2} \mathrm{OH}-\mathrm{CH}_{2} \mathrm{~S}^{-}$} & 1.518 & OPLS-AA & 1.539 & 1.540 & 1.538 & 1.543 & 1.546 & 1.548 & 1.546 & 1.551 \\
\hline & & ESP & 1.544 & 1.544 & 1.542 & 1.547 & 1.555 & 1.556 & 1.554 & 1.559 \\
\hline \multirow[t]{2}{*}{$\mathrm{MUE}^{d}$} & & OPLS-AA & $0.008 / 0.014$ & $0.009 / 0.015$ & 0.009 & 0.010 & $0.014 / 0.024$ & $0.015 / 0.025$ & 0.015 & 0.016 \\
\hline & & ESP & $0.011 / 0.016$ & $0.011 / 0.016$ & 0.011 & 0.012 & $0.014 / 0.022$ & $0.015 / 0.023$ & 0.014 & 0.015 \\
\hline \multirow[t]{2}{*}{$\mathrm{MSE}^{e}$} & & OPLS-AA & $0.005 / 0.012$ & $0.006 / 0.012$ & 0.005 & 0.007 & $0.002 / 0.014$ & $0.003 / 0.015$ & 0.002 & 0.004 \\
\hline & & ESP & $0.011 / 0.016$ & $0.011 / 0.016$ & 0.010 & 0.012 & $0.013 / 0.022$ & $0.013 / 0.022$ & 0.013 & 0.014 \\
\hline
\end{tabular}


a. The QM level is B3LYP/6-31+G*, and the MM force field is OPLS-AA. The iterative procedure for polarization was initiated by using the OPLS-AA or ESP charges for the embedded-QM calculations. The mean unsigned error (MUE) and mean signed error (MSE) were averaged over the molecules for each QM/MM treatment using the full-QM calculations as standard reference values.

b. QEq model with SCT of Rappé and Goddard

c. QEq model of Bakowies and Thiel

d. Mean unsigned error excluding/including $\mathrm{CF}_{3}-\mathrm{CH}_{2} \mathrm{OH}$

e. Mean signed error excluding/including $\mathrm{CF}_{3}-\mathrm{CH}_{2} \mathrm{O}$ 
Table S11. Full-QM and QM/MM proton affinities for the small organic molecules (with $\left.\mathrm{QM}=\mathrm{MP} 2 / 6-31+\mathrm{G}^{*}\right)^{a}$

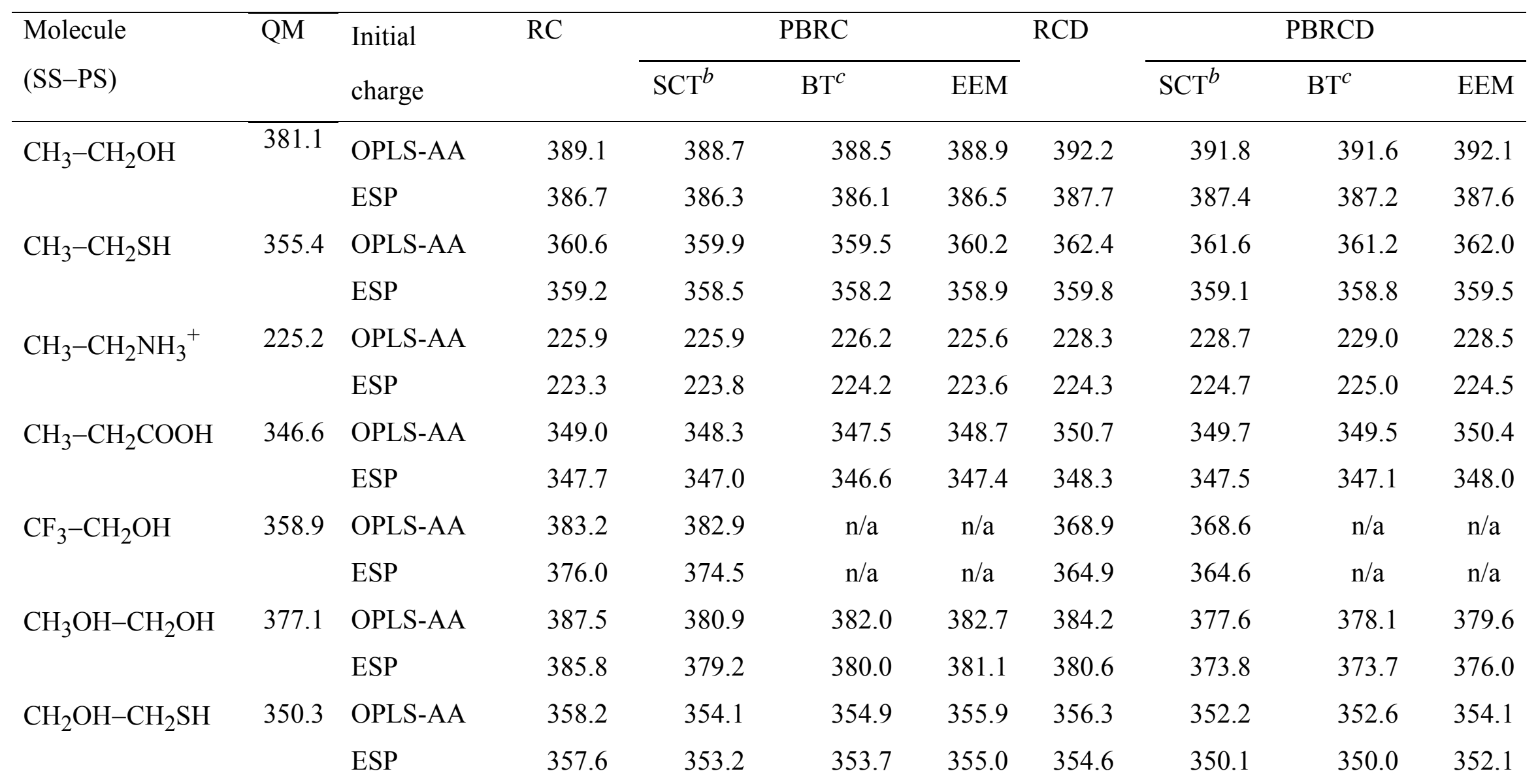




\begin{tabular}{llllllllll} 
MUE (seven) ${ }^{d}$ & OPLS-AA & $5.8 / 8.4$ & $3.7 / 6.6$ & 3.8 & 4.4 & $6.4 / 6.9$ & $4.3 / 5.1$ & 4.4 & 5.1 \\
& ESP & $4.7 / 6.5$ & $2.5 / 4.4$ & 2.5 & 3.3 & $3.6 / 3.9$ & $2.5 / 3.0$ & 2.3 & 2.6 \\
MSE (seven) & & & & & & & & & \\
& OPLS-AA & $5.8 / 8.4$ & $3.7 / 6.6$ & 3.8 & 4.4 & $6.4 / 6.9$ & $4.3 / 5.1$ & 4.4 & 5.1 \\
& ESP & $4.1 / 6.0$ & $2.1 / 4.0$ & 2.2 & 2.8 & $3.2 / 3.6$ & $1.2 / 1.8$ & 1.0 \\
\hline
\end{tabular}

a. The QM level is MP2/6-31+G*, and the MM force field is OPLS-AA. The iterative procedure for polarization was initiated by using the OPLS-AA or ESP charges for the embedded-QM calculations.

b. QEq model with SCTof Rappé and Goddard

c. QEq model of Bakowies and Thiel

d. Mean unsigned error excluding/including $\mathrm{CF}_{3}-\mathrm{CH}_{2} \mathrm{OH}$

e. Mean signed error excluding/including $\mathrm{CF}_{3}-\mathrm{CH}_{2} \mathrm{O}$ 
Table S12. QM/MM optimized Q1-M1 bond distances $(\AA)$ in comparison with full-QM results for small organic molecules $(\mathrm{QM}=$ $\left.\mathrm{MP} 2 / 6-31+\mathrm{G}^{*}\right)^{a}$

\begin{tabular}{|c|c|c|c|c|c|c|c|c|c|c|}
\hline \multirow{2}{*}{$\begin{array}{l}\text { Molecule } \\
\text { (SS-PS) }\end{array}$} & \multirow[t]{2}{*}{ QM } & \multirow{2}{*}{$\begin{array}{l}\text { Initial } \\
\text { charge }\end{array}$} & \multirow[t]{2}{*}{$\mathrm{RC}$} & \multicolumn{3}{|c|}{ PBRC } & \multirow[t]{2}{*}{ RCD } & \multicolumn{3}{|c|}{ PBRCD } \\
\hline & & & & $\mathrm{SCT}^{b}$ & $\mathrm{BT}^{\mathrm{C}}$ & EEM & & $\mathrm{SCT}^{b}$ & $\mathrm{BT}^{\mathrm{c}}$ & EEM \\
\hline \multirow[t]{2}{*}{$\mathrm{CH}_{3}-\mathrm{CH}_{2} \mathrm{OH}$} & 1.513 & OPLS-AA & 1.524 & 1.523 & 1.523 & 1.524 & 1.517 & 1.517 & 1.516 & 1.517 \\
\hline & & ESP & 1.528 & 1.528 & 1.528 & 1.528 & 1.526 & 1.526 & 1.526 & 1.526 \\
\hline \multirow[t]{2}{*}{$\mathrm{CH}_{3}-\mathrm{CH}_{2} \mathrm{SH}$} & 1.524 & OPLS-AA & 1.525 & 1.525 & 1.525 & 1.525 & 1.518 & 1.518 & 1.518 & 1.518 \\
\hline & & ESP & 1.530 & 1.530 & 1.530 & 1.530 & 1.528 & 1.528 & 1.528 & 1.528 \\
\hline \multirow[t]{2}{*}{$\mathrm{CH}_{3}-\mathrm{CH}_{2} \mathrm{NH}_{3}^{+}$} & 1.515 & OPLS-AA & 1.523 & 1.523 & 1.523 & 1.523 & 1.518 & 1.517 & 1.517 & 1.518 \\
\hline & & ESP & 1.527 & 1.527 & 1.527 & 1.527 & 1.526 & 1.525 & 1.525 & 1.525 \\
\hline \multirow[t]{2}{*}{$\mathrm{CH}_{3}-\mathrm{CH}_{2} \mathrm{COOH}$} & 1.523 & OPLS-AA & 1.527 & 1.527 & 1.527 & 1.527 & 1.521 & 1.522 & 1.521 & 1.521 \\
\hline & & ESP & 1.531 & 1.531 & 1.531 & 1.531 & 1.530 & 1.530 & 1.530 & 1.530 \\
\hline \multirow[t]{2}{*}{$\mathrm{CF}_{3}-\mathrm{CH}_{2} \mathrm{OH}$} & 1.510 & OPLS-AA & 1.552 & 1.551 & $\mathrm{n} / \mathrm{a}$ & $\mathrm{n} / \mathrm{a}$ & 1.573 & 1.572 & $\mathrm{n} / \mathrm{a}$ & $\mathrm{n} / \mathrm{a}$ \\
\hline & & ESP & 1.550 & 1.545 & $\mathrm{n} / \mathrm{a}$ & $\mathrm{n} / \mathrm{a}$ & 1.567 & 1.567 & $\mathrm{n} / \mathrm{a}$ & $\mathrm{n} / \mathrm{a}$ \\
\hline \multirow[t]{2}{*}{$\mathrm{CH}_{2} \mathrm{OH}-\mathrm{CH}_{2} \mathrm{OH}$} & 1.512 & OPLS-AA & 1.530 & 1.532 & 1.530 & 1.534 & 1.534 & 1.536 & 1.535 & 1.538 \\
\hline & & ESP & 1.533 & 1.534 & 1.532 & 1.536 & 1.540 & 1.541 & 1.539 & 1.543 \\
\hline \multirow[t]{2}{*}{$\mathrm{CH}_{2} \mathrm{OH}-\mathrm{CH}_{2} \mathrm{SH}$} & 1.516 & OPLS-AA & 1.534 & 1.535 & 1.534 & 1.536 & 1.537 & 1.539 & 1.538 & 1.540 \\
\hline & & ESP & 1.536 & 1.537 & 1.536 & 1.538 & 1.542 & 1.543 & 1.542 & 1.544 \\
\hline \multirow[t]{2}{*}{$\mathrm{CH}_{3}-\mathrm{CH}_{2} \mathrm{O}^{-}$} & 1.550 & OPLS-AA & 1.550 & 1.549 & 1.549 & 1.550 & 1.539 & 1.538 & 1.537 & 1.538 \\
\hline & & ESP & 1.559 & 1.557 & 1.557 & 1.558 & 1.555 & 1.554 & 1.553 & 1.555 \\
\hline
\end{tabular}




\begin{tabular}{lclllllllll}
$\mathrm{CH}_{3}-\mathrm{CH}_{2} \mathrm{~S}^{-}$ & 1.530 & OPLS-AA & 1.528 & 1.527 & 1.527 & 1.528 & 1.519 & 1.519 & 1.518 & 1.519 \\
& & ESP & 1.534 & 1.534 & 1.533 & 1.534 & 1.532 & 1.531 & 1.530 & 1.531 \\
$\mathrm{CH}_{3}-\mathrm{CH}_{2} \mathrm{NH}_{2}$ & 1.528 & OPLS-AA & 1.543 & 1.531 & 1.531 & 1.531 & 1.523 & 1.523 & 1.523 & 1.523 \\
& & ESP & 1.536 & 1.536 & 1.536 & 1.536 & 1.534 & 1.534 & 1.534 & 1.534 \\
$\mathrm{CH}_{3}-\mathrm{CH}_{2} \mathrm{COO}^{-}$ & 1.525 & OPLS-AA & 1.532 & 1.532 & 1.532 & 1.532 & 1.525 & 1.525 & 1.525 & 1.525 \\
& & ESP & 1.537 & 1.537 & 1.537 & 1.537 & 1.535 & 1.535 & 1.535 & 1.535 \\
$\mathrm{CF}_{3}-\mathrm{CH}_{2} \mathrm{O}^{-}$ & \multirow{2}{*}{1.532} & OPLS-AA & 1.597 & 1.596 & $\mathrm{n} / \mathrm{a}$ & $\mathrm{n} / \mathrm{a}$ & 1.644 & 1.644 & $\mathrm{n} / \mathrm{a}$ & $\mathrm{n} / \mathrm{a}$ \\
& & ESP & 1.593 & 1.592 & $\mathrm{n} / \mathrm{a}$ & $\mathrm{n} / \mathrm{a}$ & 1.630 & 1.629 & $\mathrm{n} / \mathrm{a}$ & $\mathrm{n} / \mathrm{a}$ \\
$\mathrm{CH}_{2} \mathrm{OH}-\mathrm{CH}_{2} \mathrm{O}^{-}$ & \multirow{2}{*}{1.534} & OPLS-AA & 1.563 & 1.567 & 1.565 & 1.569 & 1.573 & 1.578 & 1.576 & 1.580 \\
& & ESP & 1.569 & 1.572 & 1.570 & 1.575 & 1.585 & 1.590 & 1.588 & 1.591 \\
$\mathrm{CH}_{2} \mathrm{OH}-\mathrm{CH}_{2} \mathrm{~S}^{-}$ & \multirow{2}{*}{1.511} & OPLS-AA & 1.537 & 1.538 & 1.536 & 1.541 & 1.543 & 1.545 & 1.543 & 1.547 \\
& & ESP & 1.541 & 1.541 & 1.540 & 1.544 & 1.551 & 1.552 & 1.550 & 1.554 \\
& & & & & & & & & \\
$\mathrm{MUE}^{d}$ & & OPLS-AA & $0.012 / 0.018$ & $0.011 / 0.017$ & 0.011 & 0.012 & $0.013 / 0.024$ & $0.014 / 0.024$ & 0.013 & 0.014 \\
& & ESP & $0.015 / 0.020$ & $0.015 / 0.020$ & 0.015 & 0.016 & $0.017 / 0.025$ & $0.017 / 0.026$ & 0.017 & 0.018 \\
$M^{M S E}{ }^{2}$ & & OPLS-AA & $0.011 / 0.017$ & $0.011 / 0.017$ & 0.010 & 0.012 & $0.007 / 0.019$ & $0.008 / 0.019$ & 0.007 & 0.009 \\
& & ESP & $0.015 / 0.020$ & $0.015 / 0.020$ & 0.015 & 0.016 & $0.017 / 0.025$ & $0.017 / 0.026$ & 0.017 & 0.018 \\
\hline
\end{tabular}


a. The QM level is MP2/6-31+G*, and the MM force field is OPLS-AA. The iterative procedure for polarization was initiated by using the OPLS-AA or ESP charges for the embedded-QM calculations. The mean unsigned error (MUE) and mean signed error (MSE) were averaged over the molecules for each QM/MM treatment using the full-QM calculations as standard reference values.

b. QEq model with SCT of Rappé and Goddard

c. QEq model of Bakowies and Thiel

d. Mean unsigned error excluding/including $\mathrm{CF}_{3}-\mathrm{CH}_{2} \mathrm{OH}$

e. Mean signed error excluding/including $\mathrm{CF}_{3}-\mathrm{CH}_{2} \mathrm{O}$ 
Table S13. Full-QM and QM/MM proton affinities for amino acids (with $\left.\mathrm{QM}=\mathrm{B} 3 \mathrm{LYP} / 6-31+\mathrm{G}^{*}\right)^{a}$

\begin{tabular}{|c|c|c|c|c|c|c|c|c|c|}
\hline \multirow[t]{2}{*}{ Molecule } & \multirow[t]{2}{*}{ QM } & \multirow[t]{2}{*}{$\mathrm{RC}$} & \multicolumn{3}{|c|}{ PBRC } & \multirow[t]{2}{*}{$\mathrm{RCD}$} & \multicolumn{3}{|c|}{ PBRCD } \\
\hline & & & $\mathrm{SCT}^{b}$ & $\mathrm{BT}^{c}$ & EEM & & $\mathrm{SCT}^{b}$ & $\mathrm{BT}^{c}$ & EEM \\
\hline Ace-Lys-NMe & 229.6 & 228.2 & 229.5 & 229.5 & 228.9 & 227.3 & 228.4 & 228.1 & 228.0 \\
\hline Ace-His ${ }^{\delta}-\mathrm{NMe}$ & 245.2 & 240.8 & 248.5 & 238.8 & 248.3 & 239.7 & 247.3 & 239.2 & 247.2 \\
\hline Ace-His ${ }^{\varepsilon}-\mathrm{NMe}$ & 248.6 & 239.3 & 245.3 & 244.5 & 244.7 & 238.1 & 243.8 & 242.4 & 243.6 \\
\hline Ace-Tyr-NMe & 341.2 & 344.7 & 339.7 & 344.9 & 344.8 & 343.6 & 338.6 & 343.1 & 344.0 \\
\hline Ace-Glu-NMe & 335.2 & 335.5 & 334.6 & 335.6 & 338.6 & 334.0 & 332.8 & 333.0 & 337.2 \\
\hline $\mathrm{MUE}^{d}$ & & 3.8 & 1.8 & 2.9 & 2.9 & 4.4 & 2.6 & 3.6 & 2.7 \\
\hline MSE $e$ & & -2.3 & -0.4 & -1.3 & 1.1 & -3.4 & -1.8 & -2.8 & 0.0 \\
\hline
\end{tabular}

a. The side chain is the PS, and the backbone is the SS. The QM level is set to B3LYP/6-31+G*, and the MM force field is OPLSAA. The iterative procedure for polarization was initiated by using the OPLS-AA charges for the embedded-QM calculations. The geometries are optimized at the given QM or QM/MM level of theory, except for Ace-Lys-NMe, for which we used the geometries with QM = HF/MIDI! (in Table 4 of the paper); the side chain of Ace-Lys-NMe in the B3LYP optimized geometries bent and formed H-bonds with the backbone, making the conformations incomparable with the HF/MIDI! results. The mean unsigned error (MUE) and the mean signed error (MSE) were averaged over the molecules for each QM/MM treatment using the full-QM calculations as standard reference values. 
b. QEq model with SCT of Rappé and Goddard

c. QEq model of Bakowies and Thiel

d. Mean unsigned error

e. Mean signed error 
Table S14. QM/MM optimized Q1-M1 bond distances $(\AA)$ in comparison with full-QM results for amino acids (with QM = $\left.\mathrm{B} 3 \mathrm{LYP} / 6-31+\mathrm{G}^{*}\right)^{a}$

\begin{tabular}{|c|c|c|c|c|c|c|c|c|c|}
\hline \multirow[t]{2}{*}{ Molecule } & \multirow[t]{2}{*}{ QM } & \multirow[t]{2}{*}{$\mathrm{RC}$} & \multicolumn{3}{|c|}{ PBRC } & \multirow[t]{2}{*}{ RCD } & \multicolumn{3}{|c|}{ PBRCD } \\
\hline & & & $\mathrm{SCT}^{b}$ & $\mathrm{BT}^{C}$ & EEM & & $\mathrm{SCT}^{b}$ & $\mathrm{BT}^{c}$ & EEM \\
\hline \multicolumn{10}{|l|}{$\left(\mathrm{XH}\right.$ or $\left.\mathrm{XH}^{+}\right)$} \\
\hline Ace-His-NMe & 1.558 & 1.559 & 1.557 & 1.554 & 1.557 & 1.563 & 1.560 & 1.557 & 1.559 \\
\hline Ace-Tyr-NMe & 1.561 & 1.556 & 1.556 & 1.553 & 1.555 & 1.560 & 1.560 & 1.558 & 1.558 \\
\hline Ace-Glu-NMe & 1.552 & 1.553 & 1.552 & 1.549 & 1.551 & 1.556 & 1.555 & 1.553 & 1.554 \\
\hline \multicolumn{10}{|l|}{$\left(\mathrm{X}^{-}\right.$or $\left.\mathrm{X}\right)$} \\
\hline Ace-His ${ }^{\delta}-\mathrm{NMe}$ & 1.537 & 1.556 & 1.554 & 1.559 & 1.552 & 1.560 & 1.558 & 1.561 & 1.556 \\
\hline Ace-His ${ }^{\varepsilon}-\mathrm{NMe}$ & 1.557 & 1.561 & 1.561 & 1.558 & 1.559 & 1.566 & 1.565 & 1.563 & 1.563 \\
\hline Ace-Tyr-NMe & 1.568 & 1.564 & 1.564 & 1.562 & 1.563 & 1.570 & 1.570 & 1.569 & 1.567 \\
\hline Ace-Glu-NMe & 1.554 & 1.557 & 1.559 & 1.556 & 1.557 & 1.562 & 1.563 & 1.562 & 1.561 \\
\hline MUE $^{d}$ & & 0.005 & 0.005 & 0.007 & 0.005 & 0.007 & 0.007 & 0.006 & 0.006 \\
\hline $\mathrm{MSE}^{e}$ & & 0.003 & 0.002 & 0.001 & 0.001 & 0.007 & 0.006 & 0.005 & 0.004 \\
\hline
\end{tabular}

a. The side chain is the PS, and the backbone is the SS. The QM level is B3LYP/6-31+G*, and the MM force field is OPLS-AA. The iterative procedure for polarization was initiated by using the OPLS-AA charges for the embedded-QM calculations. If not 
otherwise indicated, only the M2 and M3 atoms in the SS were allowed to change charges. The geometries are optimized at the given QM or QM/MM level of theory, except for Ace-Lys-NMe, for which we used the geometries with QM = HF/MIDI! (in Table 4 of the paper); the side chain of Ace-Lys-NMe in the B3LYP optimized geometries bent and formed H-bonds with the backbone, making the conformations incomparable with the HF/MIDI! results. The mean unsigned error (MUE) and the mean signed error (MSE) were averaged over the molecules for each QM/MM treatment using the full-QM calculations as standard reference values. The MSE and MSE excluded the Ace-Lys-NMe molecule.

b. QEq model with SCT of Rappé and Goddard

c. QEq model of Bakowies and Thiel

d. Mean unsigned error

e. Mean signed error 
Table S15. Single-point full-QM and QM/MM proton affinities for amino acids at the full-QM optimized geometries for the amino acids (with $\left.\mathrm{QM}=\mathrm{B} 3 \mathrm{LYP} / 6-31+\mathrm{G}^{*}\right)^{a}$

\begin{tabular}{|c|c|c|c|c|c|c|c|c|c|}
\hline \multirow[t]{2}{*}{ Molecule } & \multirow[t]{2}{*}{ QM } & \multirow[t]{2}{*}{$\mathrm{RC}$} & \multicolumn{3}{|c|}{ PBRC } & \multirow[t]{2}{*}{ RCD } & \multicolumn{3}{|c|}{ PBRCD } \\
\hline & & & $\mathrm{SCT}^{b}$ & $\mathrm{BT}^{c}$ & EEM & & $\mathrm{SCT}^{b}$ & $\mathrm{BT}^{c}$ & EEM \\
\hline Ace-Lys-NMe & 229.6 & 227.7 & 228.7 & 228.8 & 227.8 & 226.8 & 227.8 & 227.5 & 227.0 \\
\hline Ace-His ${ }^{\delta}-\mathrm{NMe}$ & 245.2 & 236.6 & 243.0 & 238.7 & 241.7 & 235.6 & 241.7 & 236.7 & 240.7 \\
\hline Ace-His ${ }^{\varepsilon}-\mathrm{NMe}$ & 248.6 & 243.3 & 248.5 & 245.8 & 246.5 & 242.1 & 247.0 & 243.8 & 245.3 \\
\hline Ace-Tyr-NMe & 341.2 & 345.0 & 340.5 & 344.9 & 345.3 & 343.9 & 339.4 & 343.2 & 344.3 \\
\hline Ace-Glu-NMe & 335.2 & 337.1 & 336.2 & 336.6 & 339.8 & 335.5 & 334.3 & 334.0 & 338.3 \\
\hline MUE & & 4.3 & 1.0 & 3.0 & 3.2 & 4.4 & 1.9 & 3.7 & 3.3 \\
\hline MSE & & -2.0 & -0.6 & -1.0 & 0.3 & -3.2 & -1.9 & -2.9 & -0.8 \\
\hline
\end{tabular}

a. Single-point QM/MM calculations were carried out at the full-QM optimized geometries at B3LYP/6-31+G* level of theory, except for Ace-Lys-NMe, for which the geometry was optimized at the HF/MIDI! level of theory; the side chain of Ace-Lys-NMe in the B3LYP optimized geometries bent and formed H-bonds with the backbone, making the conformations incomparable with the HF/MIDI! results. The side chain is the PS, and the backbone is the SS. The single-point calculations were done with QM = B3LYP/6-31+G* and the MM force field of OPLS-AA. The iterative procedure for polarization was initiated by using the OPLSAA charge parameters as partial atomic charges of SS atoms in the embedded-QM calculations. The mean unsigned error (MUE) and the mean signed error (MSE) were averaged over the molecules for each QM/MM treatment using the full-QM calculations as standard reference data. 
b. QEq model with SCT of Rappé and Goddard

c. QEq model of Bakowies and Thiel 
Table S16. Full-QM and QM/MM proton affinities (kcal/mol) for amino acids (with $\left.\mathrm{QM}=\mathrm{MP} 2 / 6-31+\mathrm{G}^{*}\right)^{a}$

\begin{tabular}{|c|c|c|c|c|c|c|c|c|c|}
\hline \multirow[t]{2}{*}{ Molecule } & \multirow[t]{2}{*}{ QM } & \multirow[t]{2}{*}{$\mathrm{RC}$} & \multicolumn{3}{|c|}{ PBRC } & \multirow[t]{2}{*}{$\mathrm{RCD}$} & \multicolumn{3}{|c|}{ PBRCD } \\
\hline & & & $\mathrm{SCT}^{b}$ & $\mathrm{BT}^{c}$ & EEM & & $\mathrm{SCT}^{b}$ & $\mathrm{BT}^{C}$ & EEM \\
\hline Ace-Lys-NMe & 228.4 & 227.0 & 228.2 & 228.2 & 227.6 & 226.1 & 227.2 & 226.9 & 226.8 \\
\hline Ace-His ${ }^{\delta}-\mathrm{NMe}$ & 240.0 & 237.4 & 245.0 & 234.7 & 244.9 & 236.4 & 243.9 & 235.3 & 243.9 \\
\hline Ace-His ${ }^{\varepsilon}-\mathrm{NMe}$ & 244.2 & 235.8 & 241.6 & 240.9 & 241.1 & 234.6 & 240.2 & 238.9 & 240.0 \\
\hline Ace-Tyr-NMe & 338.9 & 343.1 & 338.0 & 343.4 & 343.0 & 342.1 & 337.0 & 341.7 & 342.3 \\
\hline Ace-Glu-NMe & 332.4 & 332.5 & 331.7 & 332.9 & 335.7 & 331.0 & 330.0 & 330.3 & 334.2 \\
\hline MUE & & 3.3 & 1.9 & 2.8 & 3.2 & 4.0 & 2.7 & 3.3 & 3.0 \\
\hline MSE & & -1.6 & 0.1 & -0.8 & 1.7 & -2.7 & -1.1 & -2.2 & 0.7 \\
\hline
\end{tabular}

a. Single-point calculations were carried out at the QM and QM/MM geometries optimized with $\mathrm{QM}=\mathrm{B} 3 \mathrm{LYP} / 6-31+\mathrm{G}^{*}$ for AceHis-NMe, Ace-Tyr-NMe, and Ace-Glu-NMe. For Ace-Lys-NMe, the single point calculations were performed at the QM and QM/MM geometries optimized with QM = HF/MIDI!. The side chain was the PS, and the backbone was the SS (see also Fig. 1). In the single-point calculations, the QM level was MP2/6-31+G*, and the MM force field was OPLS-AA. The iterative procedure for polarization was initiated by using the OPLS-AA charge parameters as partial atomic charges of SS atoms in the embeddedQM calculations. The mean unsigned error (MUE) and the mean signed error (MSE) were averaged over the molecules for each QM/MM treatment using the full-QM calculations as standard reference data.

b. QEq model with SCT of Rappé and Goddard

c. QEq model of Bakowies and Thiel 
Table S17. Single-point QM and QM/MM (with QM = MP2/6-31+G*) proton affinities (kcal/mol) for amino acids at the B3LYP/6$31+\mathrm{G}^{*}$ full-QM optimized geometries ${ }^{a}$

\begin{tabular}{|c|c|c|c|c|c|c|c|c|c|}
\hline \multirow[t]{2}{*}{ Molecule } & \multirow[t]{2}{*}{$\mathrm{QM}$} & \multirow[t]{2}{*}{$\mathrm{RC}$} & \multicolumn{3}{|c|}{ PBRC } & \multirow[t]{2}{*}{ RCD } & \multicolumn{3}{|c|}{ PBRCD } \\
\hline & & & $\mathrm{SCT}^{b}$ & $\mathrm{BT}^{c}$ & EEM & & $\mathrm{SCT}^{b}$ & $\mathrm{BT}^{c}$ & EEM \\
\hline Ace-Lys-NMe & 228.4 & 226.5 & 227.4 & 227.5 & 226.5 & 225.6 & 226.5 & 226.2 & 225.7 \\
\hline Ace-His ${ }^{\delta}-\mathrm{NMe}$ & 240.0 & 233.0 & 239.4 & 234.9 & 238.1 & 232.0 & 238.1 & 233.0 & 237.2 \\
\hline Ace-His ${ }^{\varepsilon}-\mathrm{NMe}$ & 244.2 & 239.9 & 244.9 & 242.2 & 242.9 & 238.7 & 243.6 & 240.3 & 241.9 \\
\hline Ace-Tyr-NMe & 338.9 & 343.5 & 338.9 & 343.4 & 343.6 & 342.5 & 337.7 & 341.8 & 342.7 \\
\hline Ace-Glu-NMe & 332.4 & 334.2 & 333.5 & 334.0 & 336.9 & 332.6 & 331.6 & 331.4 & 335.4 \\
\hline MUE & & 3.9 & 0.7 & 2.8 & 2.9 & 4.0 & 1.3 & 3.4 & 2.9 \\
\hline MSE & & -1.4 & 0.0 & -0.4 & 0.8 & -2.5 & -1.3 & -2.2 & -0.2 \\
\hline
\end{tabular}

a. Single-point QM/MM calculations were carried out at full-QM optimized geometries at B3LYP/6-31+G* level of theory, except for Ace-Lys-NMe, for which full-QM optimized geometries at the HF/MIDI! level of theory was used. The side chain is the PS, and the backbone is the SS (see also Fig. 1). In the single-point calculations, the QM level was MP2/6-31+G*, and the MM force field was OPLS-AA. The iterative procedure for polarization was initiated by using the OPLS-AA charge parameters as partial atomic charges of SS atoms in the embedded-QM calculations. The mean unsigned error (MUE) and the mean signed error (MSE) were averaged over the molecules for each QM/MM treatment using the full-QM calculations as standard reference data.

b. QEq model with SCT of Rappé and Goddard

c. QEq model of Bakowies and Thiel 
Table S18. QM and QM/MM (with $\mathrm{QM}=\mathrm{B} 3 \mathrm{LYP} / 6-31+\mathrm{G}^{*}$ ) atomic charges (in the unit of e) for Ace-His ${ }^{\delta}-\mathrm{NMe}^{a}$

\begin{tabular}{|c|c|c|c|c|c|c|c|c|c|}
\hline \multirow[t]{2}{*}{ Atom } & \multirow[t]{2}{*}{ QM } & \multirow[t]{2}{*}{$\mathrm{RC}$} & \multicolumn{3}{|c|}{ PBRC } & \multirow[t]{2}{*}{$\mathrm{RCD}$} & \multicolumn{3}{|c|}{ PBRCD } \\
\hline & & & $\mathrm{SCT}^{b}$ & $\mathrm{BT}^{c}$ & EEM & & $\mathrm{SCT}^{b}$ & $\mathrm{BT}^{c}$ & EEM \\
\hline \multicolumn{10}{|l|}{ PS } \\
\hline $\mathrm{C} 13$ & -0.367 & -0.596 & -0.599 & -0.795 & -0.503 & -0.536 & -0.505 & -0.751 & -0.461 \\
\hline $\mathrm{C} 14$ & 0.429 & 0.551 & 0.533 & 0.634 & 0.540 & 0.539 & 0.510 & 0.632 & 0.534 \\
\hline N15 & -0.597 & -0.658 & -0.659 & -0.829 & -0.628 & -0.650 & -0.646 & -0.793 & -0.626 \\
\hline $\mathrm{C} 16$ & -0.250 & -0.315 & -0.303 & -0.269 & -0.353 & -0.322 & -0.308 & -0.290 & -0.347 \\
\hline $\mathrm{C} 17$ & 0.222 & 0.263 & 0.273 & 0.386 & 0.227 & 0.256 & 0.252 & 0.382 & 0.225 \\
\hline N18 & -0.330 & -0.344 & -0.353 & -0.394 & -0.314 & -0.332 & -0.339 & -0.400 & -0.304 \\
\hline H19 & 0.090 & 0.158 & 0.157 & 0.189 & 0.114 & 0.152 & 0.143 & 0.190 & 0.112 \\
\hline $\mathrm{H} 20$ & 0.124 & 0.206 & 0.199 & 0.254 & 0.162 & 0.199 & 0.183 & 0.251 & 0.158 \\
\hline $\mathrm{H} 21$ & 0.196 & 0.193 & 0.190 & 0.187 & 0.202 & 0.198 & 0.194 & 0.195 & 0.201 \\
\hline $\mathrm{H} 22$ & 0.097 & 0.094 & 0.091 & 0.079 & 0.097 & 0.096 & 0.099 & 0.076 & 0.098 \\
\hline $\mathrm{H} 23$ & 0.334 & 0.349 & 0.350 & 0.359 & 0.341 & 0.348 & 0.349 & 0.364 & 0.337 \\
\hline \multicolumn{10}{|c|}{ SS } \\
\hline N7 & -0.592 & -0.500 & -0.377 & -0.953 & -0.435 & -0.547 & -0.417 & -0.971 & -0.472 \\
\hline C9 & 0.523 & 0.500 & 0.228 & 0.673 & 0.202 & 0.453 & 0.180 & 0.635 & 0.136 \\
\hline H12 & 0.049 & 0.060 & 0.049 & 0.029 & 0.032 & 0.013 & 0.002 & -0.001 & -0.020 \\
\hline $\mathrm{C} 2$ & 0.805 & 0.500 & 0.380 & 0.672 & 0.446 & 0.500 & 0.378 & 0.667 & 0.445 \\
\hline H11 & 0.333 & 0.300 & 0.279 & 0.618 & 0.222 & 0.300 & 0.274 & 0.576 & 0.221 \\
\hline $\mathrm{O} 10$ & -0.546 & -0.500 & -0.354 & -0.498 & -0.208 & -0.500 & -0.354 & -0.501 & -0.199 \\
\hline N24 & -0.517 & -0.500 & -0.345 & -0.680 & -0.399 & -0.500 & -0.344 & -0.685 & -0.393 \\
\hline $\begin{array}{c}\text { Redistributed } \\
\text { charge }\left(q_{0}\right)\end{array}$ & $\mathrm{n} / \mathrm{a}$ & 0.047 & 0.047 & 0.047 & 0.047 & 0.093 & 0.093 & 0.093 & 0.093 \\
\hline $\begin{array}{l}\text { Link atom } \\
\text { (HL) }\end{array}$ & $\mathrm{n} / \mathrm{a}$ & 0.100 & 0.122 & 0.199 & 0.114 & 0.054 & 0.067 & 0.145 & 0.075 \\
\hline
\end{tabular}

a. The side chain is the PS, and the backbone is the SS. The QM level is B3LYP/6$31+\mathrm{G}^{*}$, and the MM force field is OPLS-AA. The iterative procedure for polarization was initiated by using the OPLS-AA charges for the embedded-QM calculations. If 
not otherwise indicated, only the M2 and M3 atoms in the SS were allowed to change charges. The geometries are optimized at the given QM or QM/MM level of theory.

b. QEq model with SCT of Rappé and Goddard

c. QEq model of Bakowies and Thiel 
Table S19. Single-point QM and QM/MM (with $\mathrm{QM}=\mathrm{MP} 2 / 6-31+\mathrm{G}^{*}$ ) atomic charges for Ace-His ${ }^{\delta}$-NMe at the corresponding $\mathrm{QM}$ and $\mathrm{QM} / \mathrm{MM}$ (with $\mathrm{QM}=\mathrm{B} 3 \mathrm{LYP} / 6-31+\mathrm{G}^{*}$ ) optimized geometries $^{a}$

\begin{tabular}{|c|c|c|c|c|c|c|c|c|c|}
\hline \multirow[t]{2}{*}{ Atom } & \multirow[t]{2}{*}{ QM } & \multirow[t]{2}{*}{$\mathrm{RC}$} & \multicolumn{3}{|c|}{ PBRC } & \multirow[t]{2}{*}{$\mathrm{RCD}$} & \multicolumn{3}{|c|}{ PBRCD } \\
\hline & & & $\mathrm{SCT}^{b}$ & $\mathrm{BT}^{c}$ & EEM & & $\mathrm{SCT}^{b}$ & $\mathrm{BT}^{c}$ & EEM \\
\hline \multicolumn{10}{|c|}{ PS } \\
\hline $\mathrm{C} 13$ & -0.375 & -0.601 & -0.603 & -0.793 & -0.506 & -0.544 & -0.509 & -0.751 & -0.466 \\
\hline $\mathrm{C} 14$ & 0.426 & 0.556 & 0.539 & 0.630 & 0.545 & 0.544 & 0.515 & 0.629 & 0.540 \\
\hline $\mathrm{N} 15$ & -0.627 & -0.681 & -0.682 & -0.851 & -0.653 & -0.674 & -0.669 & -0.815 & -0.651 \\
\hline $\mathrm{C} 16$ & -0.284 & -0.363 & -0.353 & -0.316 & -0.402 & -0.368 & -0.355 & -0.337 & -0.395 \\
\hline $\mathrm{C} 17$ & 0.244 & 0.277 & 0.286 & 0.412 & 0.244 & 0.270 & 0.266 & 0.405 & 0.241 \\
\hline N18 & -0.375 & -0.379 & -0.387 & -0.431 & -0.351 & -0.370 & -0.375 & -0.437 & -0.342 \\
\hline H19 & 0.097 & 0.160 & 0.159 & 0.190 & 0.116 & 0.155 & 0.145 & 0.192 & 0.115 \\
\hline $\mathrm{H} 20$ & 0.134 & 0.211 & 0.204 & 0.254 & 0.167 & 0.205 & 0.189 & 0.253 & 0.164 \\
\hline $\mathrm{H} 21$ & 0.228 & 0.225 & 0.222 & 0.218 & 0.235 & 0.230 & 0.226 & 0.226 & 0.234 \\
\hline $\mathrm{H} 22$ & 0.120 & 0.119 & 0.117 & 0.102 & 0.122 & 0.121 & 0.125 & 0.100 & 0.122 \\
\hline $\mathrm{H} 23$ & 0.358 & 0.371 & 0.372 & 0.380 & 0.364 & 0.370 & 0.372 & 0.385 & 0.360 \\
\hline
\end{tabular}

SS

$\begin{array}{cccccccccc}\text { N7 } & -0.674 & -0.500 & -0.375 & -0.954 & -0.434 & -0.547 & -0.415 & -0.971 & -0.471 \\ \text { C9 } & 0.632 & 0.500 & 0.227 & 0.670 & 0.201 & 0.453 & 0.179 & 0.633 & 0.136 \\ \text { H12 } & 0.059 & 0.060 & 0.052 & 0.032 & 0.033 & 0.013 & 0.005 & 0.003 & -0.018 \\ \text { C2 } & 0.912 & 0.500 & 0.379 & 0.668 & 0.446 & 0.500 & 0.377 & 0.664 & 0.445 \\ \text { H11 } & 0.353 & 0.300 & 0.279 & 0.623 & 0.222 & 0.300 & 0.275 & 0.580 & 0.221 \\ \text { O10 } & -0.613 & -0.500 & -0.356 & -0.499 & -0.209 & -0.500 & -0.355 & -0.502 & -0.200 \\ \text { N24 } & -0.613 & -0.500 & -0.346 & -0.681 & -0.400 & -0.500 & -0.345 & -0.685 & -0.393\end{array}$

$\begin{array}{llllllllll}\text { Redistributed } & \mathrm{n} / \mathrm{a} & 0.047 & 0.047 & 0.047 & 0.047 & 0.093 & 0.093 & 0.093 & 0.093\end{array}$ charge $\left(q_{0}\right)$

$\begin{array}{llllllllll}\text { Link atom } & \mathrm{n} / \mathrm{a} & 0.105 & 0.126 & 0.204 & 0.118 & 0.059 & 0.071 & 0.150 & 0.079\end{array}$ (HL) 
a. The side chain is the PS, and the backbone is the SS (see also Fig. 1). QM and $\mathrm{QM} / \mathrm{MM}$ (with $\mathrm{QM}=\mathrm{B} 3 \mathrm{LYP} / 6-31+\mathrm{G}^{*}$ ) geometries were used. In the single-point calculations, the QM level was $\mathrm{MP} 2 / 6-31+\mathrm{G}^{*}$. The iterative procedure for polarization was initiated by using the OPLS-AA charge parameters as partial atomic charges of SS atoms in the embedded-QM calculations.

b. QEq model with SCT of Rappé and Goddard

c. QEq model of Bakowies and Thiel 